Revue

Revue de l'histoire des religions

de Ihistoire

des religions

1| 2017

Varia

\title{
Débat doctrinal et genre littéraire à l'époque carolingienne : les opuscules théologiques de Gottschalk d'Orbais
}

Doctrinal debate and literary genre in Carolingian times: the theological works

of Gottschalk of Orbais

\section{Warren Pezé}

\section{OpenEdition}

\section{Journals}

Édition électronique

URL : http://journals.openedition.org/rhr/8653

DOI : $10.4000 /$ rhr.8653

ISSN : 2105-2573

\section{Éditeur}

Armand Colin

\section{Édition imprimée}

Date de publication : 1 mars 2017

Pagination : 25-72

ISBN : 978-2-200-93125-4

ISSN : 0035-1423

\section{Référence électronique}

Warren Pezé, « Débat doctrinal et genre littéraire à l'époque carolingienne : les opuscules théologiques de Gottschalk d'Orbais », Revue de l'histoire des religions [En ligne], 1 | 2017, mis en ligne le 01 mars 2019, consulté le 08 janvier 2021. URL : http://journals.openedition.org/rhr/8653 ; DOI : https:// doi.org/10.4000/rhr.8653 


\section{Débat doctrinal et genre littéraire à l'époque carolingienne : les opuscules théologiques de Gottschalk d'Orbais}

Cet article entreprend l'analyse de la composition des opuscules théologiques de Gottschalk d'Orbais qu'appelait de ses vœux leur éditeur Cyrille Lambot. Ces opuscules sont composés d'une juxtaposition tantôt planifiée, tantôt accidentelle de scedulae (brouillons préparatoires, florilèges de citations bibliques et patristiques, lettres...), parmi lesquelles on trouve de véritables manuels de débat. Ces scedulae offrent une image inédite de la dissémination des écrits polémiques pendant la controverse carolingienne sur la double prédestination (années 850) et permettent de réévaluer l'écho qu'elle a rencontré dans les rangs du simple clergé.

\section{Doctrinal debate and literary genre in Carolingian times: the theological works of Gottschalk of Orbais}

This article studies the composition of the theological works of Gottschalk of Orbais. The manuscript is composed of a juxtaposition of scedulae (drafts, biblical and patristic collections, letters...), which surely reflects the will of Gottschalk in some respects, but is also the result of the transmission of his documents after his death. These papers include in particular polemical handbooks for debating. These scedulae give an unusual picture of how polemical texts circulated during the Carolingian controversy on double predestination in the 850s; they make it possible to reappraise the impact of doctrinal debate among the lower clergy. 
En 1930, à la Bibliothèque de la Bourgeoisie de Berne, dom Germain Morin fait la découverte de tout un volume d'écrits théologiques inédits qu'il attribue à Gottschalk d'Orbais (806/8-868/9)1. Le caractère exceptionnel de la découverte tient en grande partie à la place de Gottschalk dans l'histoire de l'hérésie médiévale ${ }^{2}$. Fils de comte saxon, oblat de l'abbaye de Fulda, Gottschalk est le principal protagoniste de la controverse sur la double prédestination qui secoue les royaumes carolingiens, et en particulier la Francie occidentale, pendant les années 840-870. Disputée dans neuf conciles, suscitant plusieurs dizaines de lettres et traités, elle est considérée comme la plus importante controverse du monde carolingien. Gottschalk prêche la prédestination des élus au salut et des réprouvés à la damnation - prédestination consécutive défendue par saint Augustin, mais largement éclipsée, aux premiers siècles du Moyen Âge, par une pastorale insistant sur les œuvres ${ }^{3}$.

1. Germain Morin, «Gottschalk retrouvé», Revue Bénédictine, 43, 1931, p. $303-12$.

2. Klaus Vielhaber, Gottschalk der Sachse, Bonn, 1956 («Bonner historische Forschungen », 5) ; Jean Devisse, Hincmar archevêque de Reims 845-882, Genève, Droz, 1975-1976 ; D. Ganz, "The Debate on predestination », Charles the Bald. Court and Kingdom, M. Gibson and J. Nelson dir., Hampshire, Ashgate : Variorum, 1990, p. 283-302 ; Matthew Gillis, Gottschalk of Orbais. A Study of Power and Spirituality in a Ninth-Century Life, Thèse de l'université de Virginie, 2009 ; Gottschalk and a medieval predestination controversy : texts translated from the latin, Victor Genke et Francis X. Gumerlock trad., Milwaukee, Marquette University Press, 2010 (« Mediaeval Philosophical Texts in Translation») ; Diana Stanciu, « Double Predestination, Augustinian Tradition and Carolingian Ecclesiastical Politics. The Debate that Started in the Northern Realm », Revue d'histoire ecclésiastique, 110, 2015, p. 56-102 et 619-661. On trouvera une bibliographie complète dans ma thèse, Le virus de l'erreur. Essai d'histoire sociale sur la controverse prédestinatienne à l'époque carolingienne, Université Paris I Panthéon-Sorbonne, 2014, en cours de publication dans la collection Haut Moyen Âge de Brepols.

3. Jaroslav Pelikan, La tradition chrétienne. Histoire du développement de la doctrine, t. III : Croissance de la théologie médiévale, 600-1300, Pierre Quillet trad., Paris, PUF, 1994 (1éd. Chicago, 1974), p. 85-99. Sur la prédestination augustinienne, voir Augustine through the Ages. An Encyclopedia, Allan D. Fitzgerald éd., Grand Rapids, Eerdmans, 1999, p. 391-8 (J. Patout Burns, article «Grace ») et p. 677-9 (M. Lamberigts, article «Predestination»); Augustinus Lexikon, t. III, Cornelius Mayer éd., Bâle, Schwabe AG, 2010, col. 182-242 (V. H. Drecoll, article « Gratia ») et 605-10 (T. G. Ring, article « Initium fidei ») ; Gerald Bonner, Freedom and Necessity. St. Augustine's Teaching on Divine Power and Human Freedom, Washington DC, Catholic University of America Press, 2007. 
Le moine saxon est condamné aux conciles de Mayence en 848 et Quierzy en 849, excommunié et reclus au monastère d'Hautvillers. Plusieurs parmi les meilleurs érudits carolingiens élèvent alors la voix pour défendre l'augustinisme condamné à travers Gottschalk: Ratramne de Corbie, Loup de Ferrières, Prudence de Troyes, Florus de Lyon. D'autres débats viennent bientôt s'y agréger: la vision béatifique, la nature de l'âme... Parmi ces débats, il sera souvent question dans cet article de celui qui porte sur la formule trina deitas, « déité trine », qu'Hincmar soupçonne d'hérésie et fait supprimer de l'hymne Sanctorum meritis inclyta gaudia, et que défendent Gottschalk et Ratramne ${ }^{4}$.

Le clergé du royaume de Charles le Chauve se divise, ce qui pousse le souverain à forcer le consensus : au synode de Quierzy de 853 , il contrait ses évêques à souscrire à plusieurs propositions condamnant la double prédestination. La tension atteint son point d'orgue lorsque plusieurs évêques de Lotharingie, réunis à Valence, condamnent en 855 les canons de ce synode de 853 : on se bat concile contre concile. Après l'invasion de la Francie occidentale par Louis le Germanique en 858, les clergés de Lotharingie et Francie occidentale se retrouvent au concile de Savonnières (859); après un débat houleux sur la prédestination, on décide de reporter le débat à la prochaine rencontre. Mais à Tusey, l'année suivante, la question est enterrée. Chacun impose sa doctrine et sa loi dans son royaume et l'on perd progressivement trace de la controverse sur la double prédestination, Gottschalk luimême mourant le 30 octobre 868 ou 869.

De Gottschalk, on ne connaissait, avant Morin, que deux professions de foi, les Confessiones brevior et prolixior, avec quelques fragments. La découverte du savant bénédictin devait permettre de confronter enfin la voix de «l'hérétique» à celle de ses détracteurs et d'apporter une contribution majeure à la déconstruction du discours hérésiologique médiéval. Occupé par son édition de Césaire, Morin confie celle des opuscules de Gottschalk à dom Cyrille Lambot, qui ajoute bientôt à la trouvaille de son maître celle de deux opuscules grammaticaux dans le ms. 83

4. Voir en particulier Jean Jolivet, Godescalc d'Orbais et la trinité : la méthode de la théologie à l'époque carolingienne, Paris, Vrin, 1958, et George H. Tavard, Trina Deitas. The Controversy between Hincmar and Gottschalk, Milwaukee, Marquette University Press, 1996. 
de Berne ${ }^{5}$. Retardée par la guerre, l'édition, irréprochable, ne paraît qu'en $1945^{6}$. Lambot promettait un second volume d'analyse, indispensable complément qui ne vint jamais ${ }^{7}$. Si, depuis lors, bien des travaux ont porté sur la dimension théologique des écrits de Gottschalk, le volume édité par Lambot n'a pas encore apporté sa contribution à une histoire sociale du fait controversial ${ }^{8}$. Comme l'avait souligné Lambot, le ms. 584 n'est pas composé d'une série de traités cohérents, mais d'un assemblage relativement confus de scedulae, c'est-à-dire de fiches et de fragments sur différents thèmes, que dom Lambot, pour ne pas embrouiller davantage la situation, a choisi d'éditer en suivant l'ordre du manuscrit'. L'éditeur joignait à cet avertissement un catalogue des titres rubriqués et incipit qui devait permettre au lecteur d'identifier ces scedulae ${ }^{10}$.

Il a fallu attendre la récente thèse de Matthew B. Gillis pour que l'on prenne conscience de la valeur de ce volume en termes

5. Cyrille Lambot, « Opuscules grammaticaux de Gottschalk », Revue Bénédictine, 44, 1932, p. $120-4$.

6. Euvres théologiques et grammaticales de Godescalc d'Orbais, Cyrille Lambot ed., Louvain, Spicilegium sacrum lovaniense, 1945 ( Spicilegium sacrum lovaniense », 20). Cf. François Châtillon, « Recension de Dom Cyrille Lambot, CEuvres théologiques... », Revue du Moyen Âge latin, 5, 1949, p. 255-72. Avant la publication, D. Kadner, « Aus den neuentdeckten Traktaten des mönches Gottschalk», Zeitschrift für Kirchengeschichte, 61, 1942, p. 349-58, décrit le contenu du manuscrit.

7. Lambot, Euvres théologiques, p. Ix. Cf. Pierre Verbraken, «La carrière scientifique de dom Cyrille Lambot », Revue Bénédictine, 79, 1969, p. 11-22.

8. Marie-Christine Mitterauer, Gottschalk der Sachse und seine Gegner im Praedestinationsstreit, phil. Diss., Universität Wien, 1956 ; Louise R. Gustavsson, Gottschalk Reconsidered: A Study of His Thought as It bears on His Notion of Predestination, Ph.D. diss., Yale University, 1964 ; Boyd H. Evert, Gottschalk of Orbais and the Debate over Predestination in the Ninth Century, Master Thesis, University of Dallas, 1994 ; Ariane Monteil, Le rôle de Godescalc d'Orbais dans la première controverse eucharistique, mémoire de la faculté protestante de théologie de Paris, 2002. Citons également : Ronald Hanko, « Gottschalk's Doctrine of Double Predestination », Protestant Reformed Theological Journal, 12/1, 1978, p. 31-64 ; Kurt Flasch, «Freiheit des Willens, 850-1150, », in Die Abendlandische Freiheit vom 10. zum 14. Jahrhundert, Johannes Fried ed., Sigmaringen, 1991, p. 17-49.

9. Euvres théologiques, p. XXIII.

10. Ibid., p. XIV-XV. Malgré l'avertissement de Lambot, Gillis, Gottschalk of Orbais, p. 313, considère par exemple le De praedestinatione (Euvres théologiques, p. 180-258) comme « un traité substantiel». Genke et Gumerlock, Translated texts, p. 107, sont plus prudents et parlent d'une «collection de schedulae » et d'un «matériel hétérogène». La Clavis des auteurs latins du Moyen Âge (territoire français, 735-987), t. 3, Turnhout, Brepols, 2010 (« Clavis scriptorum latinorum medii aevi », 3), p. 203-234 (notice de Jean-Paul Bouhot) a fidèlement suivi le découpage en vingt-cinq opuscules de Lambot. 
d'histoire sociale. Gottschalk, montre-t-il, diffuse dans le clergé de la province de Reims des libelles et feuillets volants, faciles à produire et transporter, souvent sans destinataire spécifique - ainsi le recueil intitué De trina Deitate ${ }^{11}$. Les Responsa de diversis, pour leur part, témoignent d'un échange épistolaire entre le moine reclus et un "jeune frère », adolescens frater ${ }^{12}$. D'autres de ces scedulae sont de véritables manuels polémiques, alimentant ses partisans en citations bibliques appropriées et en questions à poser à l'adversaire pour l'acculer dans la contradiction ${ }^{13}$. Enfin, on trouve dans certaines de ces scedulae de véritables appels à la désobéissance civile, à destination des clercs subalternes qui sympathisaient avec les thèses du moine condamné : Gottschalk argue, à cette fin, de la soumission des autorités séculières à Dieu ${ }^{14}$.

On se propose de tirer les conséquences des observations de Gillis en redécoupant les opuscules théologiques de Gottschalk dans leur intégralité. À cette fin, il faut être attentif, d'une part aux «embrayeurs » et autres marqueurs énonciatifs qui trahissent le contexte de rédaction des scedulae et permettent de les distinguer les unes des autres ${ }^{15}$; d'autre part, à l'analyse codicologique fournie par Lambot, en particulier l'index des incipit. Ce redécoupage, que l'on trouvera en annexe, s'entend comme un instrument de travail au service des historiens. Mais on entend en tirer déjà ici les leçons en matière d'histoire sociale des controverses. La nature et l'impact des débats doctrinaux carolingiens sont contestés. Selon une hypothèse minimaliste, le débat carolingien est le fait d'une mince élite lettrée et cléricale, excluant la possibilité qu'un plus vaste public de simples clercs et de laïcs ait pu constituer une opinion publique $^{16}$. Un examen approprié des scedulae de Gottschalk offre

11. Gillis, Gottschalk of Orbais, p. 282-3 et 294 (Euvres théologiques, p. 81-130).

12. Ibid., p. 293.

13. Ibid., p. 314-5 (Euvres théologiques, p. 229-31 et 238-9).

14. Ibid., p. 308-13 (CEuvres théologiques, p. 96-9, 251 et 412).

15. R. Jakobson, Shifters, verbal categories and the Russian verb, Harvard University, 1957 (repr. in Russian and Slavic Grammar, Studies 1931-1981, L. R. Waugh and M. Halle ed., Berlin, De Gruyter, 1984 ( «Janua Linguarum. Series Maior», 106), p. 41-58); Émile Benvéniste, «L'appareil formel de l'énonciation », in Problèmes de linguistique générale, II, Paris, 1974, p. 79-88 (rééd. de Langages, 17, 1970, p. 12-8).

16. Margaret Gibson, «The Continuity of Learning circa 850-circa 1050 », Viator, 6, 1975, p. 1-14 ; David E. Luscombe, «Dialectic and Rhetoric in the Ninth and Twelfth Centuries : Continuity and Change », Dialektik und Rhetorik 
un argument de poids à la thèse adverse, maximaliste ${ }^{17}$. Dans un premier temps, on décrira les plus importants de ces ensembles de scedulae en cernant le public auquel elles s'adressent. Nous en tirerons ensuite les enseignements quant à la culture du débat à l'époque carolingienne. Il sera fait référence, dans la suite de l'article, au redécoupage en annexe avec les numéros de pages de l'édition Lambot et, entre crochets, la numérotation des opuscules par Lambot. Je m'abstiens d'introduire une nouvelle numérotation : les numéros de page suffisent.

\section{Les SCEdlat de GotTschalk et LeUR PUbliC}

Dom Lambot s'est efforcé d'identifier de grandes collections ( $D e$ diversis, De Trinitate, De praedestinatione...). En l'état, celles-ci sont confuses et contiennent nombre de redites et de remplois, soit avec d'autres collections, soit avec les deux opuscules grammaticaux. Pour une part, la confusion de l'ensemble témoigne de l'histoire mouvementée des papiers personnels de "l'hérétique », dont on ignore tout, sinon qu'elle fut copiée sans nom d'auteur à Reims à la fin du IX $\mathrm{x}^{\mathrm{e}}$ siècle $^{18}$. Mais c'est aussi, pour une part, le fruit

im früheren und hohen Mittelalter. Rezeption, Überlieferung und gesellschaftliche Wirkung antiker Gelehrsamkeit vornehmlich im 9. und 12. Jahrhundert, Johannes Fried ed., Munich, Oldenbourg, 1997 ( Schriften des Historischen Kollegs, Kolloquien», 27), p. 1-20; Leidulf Melve, Inventing the Public Sphere. The Public Debate during the Investiture Contest (c. 1030-1122), Leiden, Brill, 2007 (« Brill's Studies in Intellectual History », 154), p. 50-3 ; «"Even the very laymen are chattering about it" : the Politicization of Public Opinion, 800-1200 », Viator, 44/1, 2013, p. 25-48 (p. 28-31) ; Sita Steckel, Kulturen des Lehrens im Frühund Hochmittelalter : Autorität, Wissenskonzepte und Netzwerke von Gelehrten, Cologne, Böhlau, 2011 (« Norm und Struktur », 39), p. 515-68.

17. En plus de Gillis, Gottschalk of Orbais, voir David Ganz, «Theology and the organisation of Thought », New Cambridge Medieval History, t. II, Rosamond McKitterick ed., Cambridge, Cambridge University Press, 1995, p. 758-85 ; Klaus Zechiel-Eckes, Florus von Lyon als Kirchenpolitiker und Publizist, Stuttgart, Thorbecke, 1999 ( «Quellen und Forschungen zum Recht im Mittelalter », 8) ; Florence Close, Uniformiser la foi pour unifier l'Empire. Contribution à l'histoire de la pensée politico-théologique de Charlemagne, Bruxelles, Académie royale de Belgique, 2011 («Mémoire de la classe des lettres de l'académie royale de Belgique », 59/2081).

18. Les opuscules ne portent aucun nom d'auteur dans le ms. Berne 584 (Lambot, Euvres théologiques, p. xii-xiv). Le manuscrit 83, contenant les opuscules grammaticaux, eux aussi transmis anonymement, a été également copié à la fin du $\mathrm{IX}^{\mathrm{e}}$ siècle, et non du $\mathrm{X}^{\mathrm{e}}$, comme le montre John Contreni, « The Laon Formulary and the cathedral school of Laon at the beginning of the tenth century », Learning 
de la méthode de Gottschalk. La quantité de remplois montre que le Saxon disposait d'un stock de fiches toutes prêtes, qu'il remployait dans sa correspondance selon les besoins de sa démonstration.

\section{Les Responsa de diversis}

Les Responsa de diversis sont une compilation de lettres et scedulae, adressée à un mystérieux censeur, comme l'a bien montré Jean-Paul Bouhot ${ }^{19}$. Le censeur est un supérieur (abbé ou évêque, sans doute) de Gottschalk, qui semble bien le connaître (et me habeatis deinceps fideliorem...), avec qui il semble avoir correspondu (de omnibus quae recolere potestis memoria laudabili...); il le prie de le corriger en annotant directement le livret (istis in quaternionibus id inserentes). La compilation est précédée d'une table des matières qui correspond très imparfaitement au contenu, qui n'est d'ailleurs pas numéroté, ce qui rend malaisé de faire correspondre les textes à ladite table des matières ${ }^{20}$. Le dix-huitième texte numéroté par $\mathrm{C}$. Lambot dit répondre à «la première question» d'un correspondant, ce qui montre bien que Gottschalk a rassemblé pour son censeur une série de papiers d'origines diverses ${ }^{21}$.

Les raisons qui ont poussé Gottschalk à compiler toute une série de scedulae, d'y accoler un sommaire et de les adresser à un

and Culture in Carolingian Europe. Letters, Numbers, Exegesis and Manuscripts, Hampshire, Ashgate : Variorum, 2011, p. 1-14 (1-5, sur les liens entre Laon et Reims). Le nom du prévôt de l'abbaye de Rébais, mentionné par Gottschalk p. 170, a été gratté dans le manuscrit (f. 51v), ce qui semble indiquer que c'était encore un sujet sensible pour les moines de Reims un demi-siècle plus tard. On trouve un obèle dans la marge, signe critique désignant les passages corrompus.

19. Jean-Paul Bouhot, Ratramne de Corbie, Histoire littéraire et controverses doctrinales, Paris, Études augustiniennes, 1976, p. 43-5. Cf. Lambot, Euvres théologiques, p. 131-2.

20. Comparer par exemple les titres [xxviii-xxi] (Lambot, Euvres théologiques, p. 131) au texte afférent (p. 171-9).

21. Ibid., p. 171 : Noveris care primam profundam abtidam quin ut omnino scis arduam quaestionem tuam... La question que Gottschalk désigne ici comme la « première » est, dans le sommaire p. 131, la dix-huitième. Que Gottschalk soit l'auteur du sommaire ne fait pas de doute, au vu de certaines formulations : De trina deitate et una, quae est sicut firmissime teneo et veraciter credo trina in personis, una in deitate substantiae, vel in unitate naturae [...] absint insta, vos autem respondete rectiora. Gillis, Gottschalk of Orbais, p. 293, note 44, estime que la table des matières est le questionnaire du correspondant de Gottschalk; l'argumentation de Bouhot (non cité par Gillis) est plus convaincante. 
personnage puissant sont encore largement hypothétiques, mais ont sans doute un lien avec les débats sur la nature de l'âme à la cour de Charles le Chauve dans les années $850^{22}$. C'est l'étape antérieure, c'est-à-dire les différentes lettres qui composent le recueil, qui nous intéresse ici $^{23}$. Le destinataire de la plupart des lettres est un homme que Gottschalk tutoie, à la différence du censeur qu'il vouvoie. Cet homme l'a consulté sur plusieurs questions : les scedulae sont des réponses, soit de remploi, soit rédigées pour l'occasion ${ }^{24}$. Il s'agit d'une personne avec qui Gottschalk a de la familiarité; le Saxon prononce pour lui une prière et parle de lui comme d'un jeune frère (adolescens frater) ${ }^{25}$. Cette unanimité se traduit par l'emploi occasionnel de la première personne du pluriel.

À certains moments, l'énonciation passe du tutoiement au vouvoiement. Cela signifie-t-il que Gottschalk a changé de correspondant? Non: ses correspondants sont seulement plus nombreux. On s'en aperçoit en particulier dans une séquence de trois scedulae, rédigées l'une après l'autre à quelques jours de distance. Dans la première (p. 162-4), Gottschalk répond à une question posée par son interlocuteur: est-ce que les prophètes païens ont reçu le don de l'Esprit Saint? Selon le Saxon, les réprouvés peuvent être touchés par l'Esprit sans pour autant le recevoir. Quelques jours plus tard (nuper, écrit-il), il reprend la plume pour partager une nouvelle idée (p. 164-5) : les prophètes antiques peuvent être mus par les démons, à l'instar de la prophétesse en Ac, 16, 16-8. Cette fois, il utilise le vouvoiement ; et promet d'envoyer bientôt un texte plus détaillé à l'aide du commentaire de l'Ambrosiaster sur Paul. Plusieurs jours plus tard, il expédie la scedula promise (p. 166-71). Il s'excuse de s'être fait désirer, toujours en vouvoyant; on lui a soustrait le volume

22. Bouhot, Ratramne, p. 43-5.

23. Gillis, Gottschalk of Orbais, p. 293, a fourni une analyse du contenu de ces Responsa; tout en remarquant fort justement qu'il s'agit d'un recueil de lettres échangées avec des moines, il confond l'adolescens frater (p. 149-50) avec le censeur anonyme (que Gottschalk vouvoie).

24. C'est le cas, semble-t-il, des chapitres 1,2 et 5 qui revêtent, dans le sommaire, la forme d'une question correspondant parfaitement au contenu. Gottschalk affirme dans le chapitre 1, p. 132, que son correspondant lui a posé plusieurs questions : quaestiones tuas.

25. Ibid., p. 149-50. Cela contredit la thèse de Gillis que Gottschalk n'a pas de relation personnelle avec son correspondant (Gottschalk of Orbais, p. 294). 
de l'Ambrosiaster qu'il convoitait ${ }^{26}$. Il a dû se rabattre sur le commentaire de Bède sur les Actes des Apôtres.

Ici, Gottschalk s'adresse à un moine et, à travers lui, aux frères qui l'entourent. Il connaît ces personnes et se permet des confidences: dans la troisième scedula citée (p. 169-70), on trouve le récit, tiré des pérégrinations de Gottschalk dans les Balkans (années 840), d'une bataille entre le duc croate Trpimir et les Byzantins en Dalmatie; une anecdote sur un médecin de Spolète venu à Rébais; une anecdote également sur la vision de Wettin de Reichenau - monastère où Gottschalk avait étudié dans les années 820. Ces expériences personnelles, inutilisables en controverse, montrent que la consultation n'a pas d'arrièreplan polémique. Au terme de ce dernier courrier, Gottschalk peut écrire à ses correspondants : «je pense que vous avez maintenant un commentaire bien suffisant de la question que vous m'avez proposée il y a maintenant quelque temps $»^{27}$. Cet échange de textes courts, à plusieurs jours d'intervalle, illustre de manière concrète la relation entretenue par Gottschalk avec un groupe de moines. Ils le consultent et, malgré les vissicitudes de sa réclusion, il leur fait parvenir ses réponses.

Pour le Saxon, une lecture peu soigneuse de la Bible et des Pères est l'origine de l'hérésie, ce qui l'amène à donner des instructions de lecture à ses disciples ${ }^{28}$. Il recommande à un ou plusieurs destinataires des scedulae des Responsa de diversis une série d'ouvrages ${ }^{29}$. La plupart sont les textes les plus commentés de la

26. Ibid., p. 166.

27. Ibid., p. 171.

28. Ibid., p. 269 : Ne quaeso frater indigneris quod ut ea quae de deo dicuntur diligenter et intellegenter legas frequenter et humiliter admoneris, quia multos malo voto vides per peccatum legere et ob id consequenter prout merentur propter poenam peccati male coguntur intellegere.

29. Ibid., p. 146: Lege primum librum Retractationum, tunc enim cernes atque discernes [...] inspice Aethimologiam [...] p. 151 : Unde si plura vis nosse, Enchyridion et Ypommisticon sancti Augustini lege et tres libros ipsius De libero arbitrio et duas eius epistolas ad Valentinum et librum De gratia et libero arbitrio [...] et librum De correptione et gratia et omelias eius In evangelium secundum Iohannem [...] p. 152: Lege si vis etiam In canticum canticorum ultimum librum Bedae. [...] Lege Expositionem beati Ambrosii in epistolam ad Romanos et Iohannis Chrisostomi De alligatione asinae et canones ducentorum quatuordecim episcoporum quorum unus fuit sanctus Augustinus contra heresim Pelagii et Caelestii et tres epistolas Afrorum episcoporum ad papam Innocentium et tres eiusdem ad eosdem atque decreta Caelestini papae ad omnes episcopos 
querelle, à savoir: les traités d'Augustin sur la grâce et le librearbitre, avec les Retractationes ${ }^{30}$, l'Enchiridion, l'ultima pars de la Cité de Dieu $^{31}$, la plupart des monuments de la controverse sur la grâce du v viècle, c'est-à-dire les actes du concile de Carthage de 418 , les lettres des évêques d'Afrique à Innocent $\mathrm{I}^{\mathrm{er}}$ et ses réponses ${ }^{32}$, les décrétales de Célestin aux évêques de Gaule ${ }^{33}$, les traités de Prosper d'Aquitaine et, enfin, les traités de Fulgence. Il recommande également des œuvres moins directement liées à la prédestination mais pourtant citées par les controversistes : la dernière partie des Moralia in Job de Grégoire le Grand ${ }^{34}$, ses homélies sur Ézechiel ${ }^{35}$, Le commentaire sur les Actes des apôtres de Bède ${ }^{36}$, le commentaire de l'Ambrosiaster sur l'épître aux Romains ${ }^{37}$, le commentaire

Galliae, et Casiodorum In psalmos [...] et librum Prosperi contra conlationem Casiani quae praenotatur De protectione dei et Responsiones eius ad Gallos Genuenses et Vincentinos et eius Epistolam quae est in capita ad Rufinum et ultimam partem Moralium sancti Gregorii et primam In Hiezechihelem [...] lege in Enchyridion totum capitulum sub titulo De amissione liberi arbitrii... p. 155 : lege librum sancti Fulgentii... p. 163 : [...] lege sanctum Augustinum in secunda parte De civitate dei [...] quaeso quod legas Bedae Commentum in actus apostolorum ubiubi quiuerit reperiri, etc. - Je ne commente, dans les notes suivantes, que les œuvres dont le rapport avec la double prédestination est le moins évident.

30. Citées par Ratramne (PL 121, col. 68), Prudence (PL 115, passim), Loup (PL 119, col. 661), Florus (Liber de tribus epistolis, in Flori Lugdunensis opera polemica, Klaus Zechiel-Eckes et Erwin Frauenknecht ed., Turnhout, Brepols, 2014 (« Corpus christianorum continuatio mediaevalis » [CCCM] 260), p. 453-4) Hincmar (PL 125, passim).

31. La Cité de Dieu était souvent répartie en deux tomes (I-XV, XVI-XXII). On trouve dans le dernier livre une référence à la prédestination au châtiment qui n'a pas échappé, par exemple, à Loup de Ferrières (PL 119, col. 648).

32. Citées plusieurs fois par Prudence (PL 115, col. 1073, 1077, 1115).

33. Dont Florus a réalisé une compilation préservée dans le ms. Gand, UB, 249 ; voir Pierre Chambert-Protat, «Le travail de Florus de Lyon sur la prédestination: un état de la documentation conservée. Avec un dossier d'extraits patristiques resté inédit », La controverse carolingienne sur la double prédestination : histoire, textes, manuscrits, actes du colloque des 11-12/10/2013, id., Jérémy Delmulle, Warren Pezé et Jeremy C. Thompson ed., Paris, Études Augustiniennes, à paraître.

34. On sait que les Moralia étaient découpées en tranches de cinq livres. La dernière partie comprend les derniers livres à partir du vingt-septième. Ils sont fréquemment cités, dès Ratramne, dont on a conservé le manuscrit personnel : Paris, BNF, latin 12248.

35. Citées par Prudence (PL 115, en particulier col. 1183-5), Loup (PL 119, col. 662), Hincmar (PL 125, passim).

36. Hincmar, PL 125, col. 300 et passim.

37. Cité en particulier par Prudence (PL 115, col. 1185) et Hincmar (PL 125, passim). 
de Cassiodore sur les psaumes ${ }^{38} \ldots$ Quasiment toutes les œuvres conseillées par Gottschalk à son correspondant sont parmi les plus citées des controversistes. Mieux : il met son lecteur en mesure de retrouver le manuscrit d'un auteur dont le nom lui échappe, en précisant qu'il l'a lu jadis à Orbais; que le manuscrit contient une compilation dont il a oublié le détail ; et que, fort ancien, il est relié de cuir noir ${ }^{39}$. Le correspondant était, sinon moine d'Orbais, du moins en mesure de s'y rendre pour retrouver le codex.

\section{Le De praedestinatione}

Les fragments réunis sous le titre De praedestinatione (p. 180-258), constituant ce qui semble être le plus long «traité » de Gottschalk, inspirent des réflexions de même nature. La compilation est encadrée, dans le manuscrit, par le titre $D e$ praedestinatione et par un explicit de praedestinatione (p. 180 et 258$)^{40}$. Il est impossible de savoir si ces titres relèvent de l'initiative des copistes ou bien de Gottschalk. En revanche, il est sûr que cette compilation a été au moins partiellement constituée par le Saxon lui-même. On trouve en son centre une exhortation au lecteur, au ton conclusif (p. 212-7), qui porte sur des aspects techniques de la controverse sur la prédestination : la volonté de salut restreinte (Dieu a-t-il voulu le salut des réprouvés, comme le laisse penser I Tim 2, 4, ou n'a-t-il voulu le salut que de ceux qu'il sauve réellement?) et la rédemption des réprouvés (dans quelle mesure peut-on parler de rédemption pour ceux que Dieu ne sauve pas ?). Cette exhortation fait référence « aux témoignages ci-dessus » (p. 216), montrant qu'il y a là un florilège cohérent. La difficulté est de savoir jusqu'où ce «ci-dessus » remonte : jusqu'au titre De praedestinatione (p. 180) ? C'est peu probable vu le nombre de textes ne concernant en rien la prédestination (Trinité, grammaire...) qui s'y sont glissés (p. 190-1 [10], 193-4 [12],

38. Lui aussi cité par bien des controversistes : Ratramne (PL 121, col. 55), Prudence (PL 115, en particulier la chaîne de citations col. 1310, et passim), Hincmar (PL 125, col. 145 et passim).

39. Lambot, Euvres théologiques, p. 175 : nomen ipsius Orbacis olim legi in quodam uetusto libello cum ipso illius opusculo, inter cetera multa quorum tamen nulla plus recolo, qui libellus nigra prorsus est pelle contectus.

40. Dans le manuscrit, le titre se trouve au pied de la page précédant l'incipit, f. $56 \mathrm{v}$. 
194-6 [13], 196 [14], 197 [15]). Ou bien jusqu'à la série d'extraits et de témoignages sur la prédestination commençant plus bas, p. 197 [VIII] - hypothèse la plus probable ? À moins que le «ci-dessus» ne concerne que la courte compilation précédant le passage conclusif (p. 209-12 [VIII, 7-X, 1]) ? De même, les scedulae suivantes [XI-XVIII] se rattachent, pour la plupart, au thème de ce passage central, c'est-à-dire la rédemption des réprouvés, et reflètent sans nul doute une compilation intentionnelle de Gottschalk; mais à cette compilation sont venus se mêler, à cause de l'histoire chaotique du dossier, des feuillets qui ne concernent en rien la prédestination (voir surtout p. 245-7). Il faut, ici aussi, nous concentrer sur les scedulae compilées par le Saxon et reflétant une étape antérieure de son travail.

Celles-ci sont adressées à plusieurs individus et à leur entourage. Il y a des remplois : Gottschalk n'aurait pas adressé les mêmes textes aux mêmes personnes dans la même compilation. Le tutoiement indique que les destinataires sont aussi proches de Gottschalk que ceux des scedulae composant les Responsa de diversis. Le Saxon dit tantôt «cher fils", tantôt «frère ». Le destinataire mérite, lui aussi, des anecdotes sur le passé de Gottschalk, en particulier une référence au séjour en Dalmatie dans les années 840 (p. 208). Des références au chant liturgique laissent discerner un moine entouré de sa communautét ${ }^{41}$. Gottschalk propose les problèmes et leurs solutions à son correspondant, qu'il s'agisse de la Trinité, de grammaire ou de liturgie ${ }^{42}$. Il a déjà écrit à son destinataire ${ }^{43}$. On trouve des références à des écrits perdus de sa plume que connaît son lecteur ${ }^{44}$.

41. Ibid., p. 181 : illa quam de sancta cruce antiphona debita veneratione cantatis...; p. 195 : inde male fecit qui hoc ultimum testimonium in propheta depravando corrupit, vos autem bene feceritis id corrigendo; p. 232 : notissimum vobis est...

42. Ibid., p. 193 : Ni post pharisaea convivia superfuisset cenae, nihil prorsus hinc a me tibi quaestionis proponeretur quia scilicet nihil esset quod exponeretur. Manet ergo nunc quaestio quae per iunctionem seu reiunctionem sui soluitur... Cf. les parallèles avec les opuscules grammaticaux ; par exemple p. 190 et 422 ou p. 180 et 479 .

43. Ibid., p. 250 : sicut facile reminisci potes... Voir aussi p. 194.

44. Ibid.,p. 237 (opuscule 14) : Quantum, inquam, differant inter se hae salutes duae, meministi me prout puto luce clarius alibi dixisse decreuisse distinxisse. 


\section{La compilation De Trinitate}

La compilation intitulée par Lambot [De trinitate, XIX] combat la thèse que trois Esprits ontologiquement distincts coexistent en la Trinité, et défend la thèse contraire que l'Esprit Saint est un et trine. Elle se compose d'une longue disputatio (p. 260) qui a sa propre conclusion (p. 271), puis d'une série d'excerpta de la lettre 238 d'Augustin. La cohérence est assurée par la conclusion où il est fait référence aux excerpta et « à tout ce qui les précède », et où le thème est rappelé. L'ensemble est adressé à un « très cher frère » (p. 278), visiblement proche de Gottschalk ${ }^{45}$. Cet ami lui a adressé une question portant sur un passage du Commentaire sur l'épître aux Galates de Jérôme affirmant que la Trinité est composée de trois Esprits ${ }^{46}$.

Dans le détail, la disputatio se compose de feuillets écrits l'un après l'autre et assemblés en liasse, comme le montre un feuillet sur le baptême, totalement hors-sujet, qui s'est glissé au milieu d'eux par mégarde (p. 262-4). Elle est placée sous le signe de la surenchère. Chaque fois qu'il conclut l'un de ses textes, ainsi après un premier feuillet: sufficit deo gratias, amen (p. 260), Gottschalk reprend bientôt la plume pour rajouter des preuves, de crainte que le passage précédent ne suffise pas (si... non sufficiat, p. 260 ; quod si putas nimium, p. 261...). Il craint que la thèse des trois Esprits ne se répande parmi les simples moines ou qu'une polémique n'éclate - il est pourtant clair que celle-ci n'a pas éclaté $^{47}$. Dans ces années de polémique généralisée, la psychose de la controverse est, ici, évidente. Après avoir conclu sa disputatio sur un dernier message au frater, Gottschalk ajoute une adresse à «tout lecteur» (quisquis haec legerit..., p. 272) ouvrant la série d'extraits de la lettre 238 d'Augustin. Cette énonciation ouverte se retrouve dans la conclusion générale, p. 278. On voit ici comment Gottschalk non seulement ouvre sa réponse à un public plus large,

45. Cf. ibid. p. 266 : proinde germaniter precor pariter et hortor ut nullo modo fastidias medullitus audire quae loquor, quia prorsus amore veritatis atque fraternae caritatis tam multifarie, ne non intellegatur, quae nimis necessaria conspicor haec supradictis adicere compellor.

46. Ibid., p. 259.

47. Ibid., p. 261: quod si putas nimium, multi tamen sunt alii quibus consulendum censui, ne sibi faterentur esse modicum; p. 267 : praeterea si post haec omnia, vel ipse cogitaveris vel tibi forte dixerit aliquis ideo tres esse in trinitate spiritus etc. Cette dernière phrase montre bien qu'il n'y a pas de conflit. 
mais aussi transforme la consultation de son correspondant en polémique contre des détracteurs putatifs - sans raison apparente. La dimension hérésiologique est évidente ${ }^{48}$.

\section{Autres opuscules de Gottschalk}

Les deux scedulae et la collection patristique sur l'âme [Quaestiones de anima, XXI] (p. 283-91) sont aussi le reflet du public élargi de Gottschalk. Celui-ci dresse un bilan de la réflexion patristique sur l'épineuse question de la transmission de l'âme avant de proposer sa propre solution ${ }^{49}$. Il s'adresse à toute une communauté, en particulier dans un passage aux accents protoromans :

Je vous prie pourtant, à cause du Seigneur, que vous teniez secret cet écrit chez vous, pour aussi longtemps que je serai dans cet état, parce que cela fait six ans cette année que ce fait m'a été manifesté par le seigneur Dieu, et j'ai fait le vœu d'en écrire un livre, pour autant que cela lui plairait, avec son aide et sa commisération ${ }^{50}$.

La scedula de seminibus animatis est adressée à un ami à qui il a déjà écrit sur cette question ${ }^{51}$. La plupart des scedulae et fragments compris dans la compilation artificielle qui suit [De diversis, XXIII] ont des destinataires différents, tantôt une communauté (p. 299), tantôt un ami et son entourage (p. 312-3). Le De corpore et sanguine Domini est un traité rédigé contre le traité de même titre de Paschase Radbert, connu aussi par un manuscrit postérieur d'Hériger de Lobbes. Les opuscules théologiques s'achèvent avec plusieurs scedulae sur la prédestination et la rédemption (p. 338-46).

\section{Les correspondants de Gottschalk}

Concluons sur la question du public. La majorité des opuscules de Gottschalk ne sont pas, à l'exception du De corpore et sanguine

48. Ibid., p. 278: Arbitror frater karissime non modo tibi uerum cunctis etiam minus capacibus, non quidem duris et contentiosis hereticis sed mitibus et humilibus corde catholicis et orthodoxis...

49. Cf. à ce sujet Gérard Mathon, L'anthropologie chrétienne en Occident de saint Augustin à Jean Scot Érigène, Lille, Faculté de théologie, 1964 (thèse dactylogaphiée, utilement résumée dans «Les attitudes de quelques théologiens carolingiens en face des indéterminations augustiniennes en matière d'anthropologie », Mélanges de science religieuse, 22/1, 1965, p. 33-44).

50. Lambot, Euvres théologiques, p. 290 (remarquer habeo factum votum).

51. Ibid., p. 291 : uel uti recordaris et scripsi. 
Domini, des traités à la manière de Ratramne ou Prudence, mais des compilations de scedulae, de brouillons remployés, de collections de citations bibliques et patristiques, assorties de développements propres et, parfois, de passages «architectoniques » assurant leur cohésion. Ces compilations sont pour la plupart adressées à des moines proches du reclus et à leur entourage direct. Dans le De praedestinatione, le Saxon écrit: "l'antienne que vous chantez... $»^{52}$. À nouveau, dans l'opuscule De trinitate, il fait référence au chant monastique pratiqué par son destinataire ${ }^{53}$. Dans les opuscules grammaticaux, il écrit que son destinataire entend la messe tous les jours ${ }^{54}$. Les références à la pratique liturgique trahissent un milieu monastique.

Ces correspondants étaient-ils d'Hautvillers, ou d'autres monastères ? C'est une question difficile. "L'ami » auquel sont adressés les Responsa de diversis peut-être originaire d'Hautvillers ou d'Orbais. Gottschalk écrit «ici» au lieu du nom de son monastère et il décrit, on l'a vu, un livre se trouvant à la bibliothèque d'Orbais ${ }^{55}$. De même, le destinataire des textes composant le De praedestinatione doit comprendre des allusions à l'abbé Halduin d'Hautvillers, déposé en $853^{56}$. Cela étant, Gottschalk n'écrit pas toujours aux mêmes personnes. Il n'aurait pas remployé trois fois le même extrait avec le même correspondant ${ }^{57}$. On trouve un même passage sur les syllogismes dans les Responsa de diversis, la scedula De syllogismis de la compilation De praedestinatione, et chacun des deux opuscules grammaticaux ${ }^{58}$ ! S'il ne s'agit pas de moines d'Hautvillers, ils peuvent tout du moins venir de

52. Lambot, Euvres théologiques, p. 181.

53. Ibid., p. 266 : quemadmodum canis etiam crebro [...] frequenter etiam per singulos dies immo cursus [NB : heures canoniales] audis...

54. Ibid., p. 458 : quod audis ad missam cotidie.

55. Ibid., p. 156.

56. Ibid., p. 204-6: Gottschalk remploie tout un paragraphe déjà cité p. 180, sans y faire aucunement allusion, ce qui montre l'absence d'unité du $D e$ praedestinatione tel qu'édité par Lambot. Il est fait référence ici à un corrector immo corruptor qui est certainement Halduin d'Hautvillers, déjà vilipendé pour ses corrections liturgiques dans les Responsa de diversis (p. 142). Une autre allusion à une correction indue, cette fois de la Bible, se trouve p. 195. Sur la déposition d'Halduin, voir Martina Stratmann, Hinkmar als Verwalter von Bistum und Kirchenprovinz, Sigmaringen, Thorbecke, 1991 («Quellen und Forschungen zum Recht im Mittelalter », 6), p. 57.

57. Par exemple, ibid., p. 301-2, 313-4 et 478-9.

58. Ibid., p. 155-6, 206-7, 419 et 459-60. 
monastères où Gottschalk a séjourné, comme Orbais ou Rébais - la liste pouvant sans doute être allongée : il y a dix abbayes et couvents dans le diocèse de Reims du temps d'Hincmar ${ }^{59}$. En somme, les œuvres éditées par dom Lambot révèlent la correspondance quotidienne que Gottschalk entretenait avec les moines d'Hautvillers et des monastères environnants ; elles montrent aussi que par l'intermédiaires d'amis, Gottschalk pouvait transmettre à des communautés entières de courts textes sur la Trinité ou la prédestination.

\section{Des Manuels de polémiQue}

La correspondance entre Gottschalk et ses soutiens fait la part belle au débat. Il relate au destinataire des Responsa de diversis une discussion avec l'abbé Halduin d'Hautvillers venu le visiter dans sa cellule ${ }^{60}$. Ce dernier répète la thèse du notaire royal Énée, inspiré de Jean Scot Érigène, que l'on ne peut interpréter Augustin qu'avec l'aide de la dialectique ${ }^{61}$. Gottschalk, exaspéré qu'on lui répète l'argumentation de l'Érigène comme un perroquet, cite un passage du commentaire de Jérôme sur l'épître aux Galates où l'auteur applique un syllogisme à un passage de saint Paul ; il montre ainsi sa maîtrise de la logique et, à le croire, laisse Halduin bouche bée $^{62}$. De même, il rapporte un débat succédant à une assemblée rémoise de date inconnue : Gottschalk y réfute les arguments d'un adversaire anonyme qui affirme que l'Esprit Saint a du sang ${ }^{63}$. Il lui donne le surnom de Faustus manicheus, célèbre adversaire de saint Augustin ; comme dans le cas de l'opuscule [De Trinitate, XIX], la dynamique hérésiologique joue à plein. Gottschalk transcrit enfin, dans l'opuscule De trina deitate, une question qu'il affirme

59. Martina Stratmann, Hinkmar von Reims, p. 57.

60. Lambot, Euvres théologiques, p. 156-7.

61. Sur le notaire Énée, voir Georges Tessier, Recueil des actes de Charles II le Chauve roi de France, III, Paris, Imprimerie nationale, 1955, p. 60-4.

62. Le Commentaire de Jérôme sur l'épître aux Galates est souvent cité pendant la controverse prédestinatienne. Plusieurs notes du cercle de Prudence dans le ms. Cambridge Mass., Houghton Library, fMS Typ 495 insistent sur la dialectique (f. 74r, 98r); cf. Warren Pezé, «Deux manuscrits personnels de Prudence de Troyes », Revue Bénédictine, 124, 2014, p. 114-50.

63. Lambot, Euvres théologiques, p. 298. 
«avoir l'habitude de poser souvent, lorsqu' [il] débat $»^{64}$. Tous ces textes campent Gottschalk en virtuose du débat, virtuosité, nous le verrons plus loin, qu'il n'usurpe pas.

Ces récits de dialogues peuvent être comparés à un autre genre de documents qu'il fait circuler: des manuels de polémique. Ces manuels rédigés sur de courtes scedulae devaient servir de support dans les discussions. Elles montrent à quel point la culture du débat avait pénétré profondément dans le cloître. En voici quelques exemples. L'opuscule [XII] de Lambot, consacré à la question très discutée de la rédemption des réprouvés, est une courte scedula de quatre pages repérée par Gillis. Elle revêt la forme d'un interrogatoire fictif : le lecteur de Gottschalk est invité à appliquer ce manuel dans une situation de débat avec un contradicteur. C'est un précieux témoin de la pénétration du débat doctrinal dans la culture monastique. Une véritable maïeutique de controverse y est à l'œuvre. Elle est bâtie sur une habile alternance de questions et d'objections fondées chaque fois sur une citation biblique. À la fin du questionnement, le contradicteur est contraint d'accepter, à la lumière du cas des réprouvés baptisés, et après un dédale de citations, que le baptême ne fait pas participer à la passion rédemptrice :

J'aimerais pourtant ajouter ceci, pour donner un bon conseil à cet infâme vaurien : auquel des deux mondes du juste jugement de Dieu pense-t-il qu'appartiennent les réprouvés baptisés; au monde réconcilié et purifié, ou au monde ennemi, accusé et condamné ? S'il dit qu'ils appartiennent au monde réconcilié, sauvé et purifié, dis-lui : «Alors, ils sont réconciliés, sauvés et purifiés par la mort du fils de Dieu et du coup, ils seront sauvés par lui de la colère $(\operatorname{Rm} 5,9)$ »? $\mathrm{Si}$, bon gré mal gré, il reconnaît que c'est faux et qu'il répond qu'ils appartiennent au monde ennemi, accusé et condamné, pousse-le dans ses retranchements et réfute-le en toute confiance, avec le sourire : « Eh bien, que tu veuilles ou non, tu es enfin forcé de croire, comprendre et confesser que le Christ, fils de Dieu, n'a pas racheté les réprouvés baptisés par son sang, et ne les a ni réconciliés, ni sauvés, ni purifiés ${ }^{65}$.

64. Ibid., p. 86-7 : Unde crebro sic solebam et soleo dicere disputando...

65. Ibid., p. 230 : Addere tamen et istud uolo prudenter illum nequissimum nebulonem consulendo, ad quem duorum mundorum reprobos baptizatos pertinere putet iusto dei iudicio, utrum uidelicet ad mundum reconciliatum atque mundatum an ad mundum inimicum damnatum inquinatum. Si pertinere dixerit eos ad mundum reconciliatum saluatum atque mundatum, dices ei: Ergo reconciliati saluati atque mundati sunt per mortem filii dei et ideo salui erunt ab ira per ipsum ? Quod si uolens nolens uiderit atque dixerit non esse uerum ac per hoc responderit eos pertinere potius ad mundum inimicum damnatum inquinatum, infer ipse consequenter et eum coartando confuta dicens hilariter atque fidenter : Igitur 
Le dialogue se répète dans l'opuscule De reprobis baptizatis, adressé à un ami, qu'a aussi repéré Gillis. Après l'avoir averti des passages bibliques qu'il doit opposer à ses adversaires ${ }^{66}$, Il dresse un interrogatoire fictif. S'ils affirment que Dieu ne sauve que ceux qui le veulent, il faut leur demander : pourquoi Dieu sauve-t-il des nouveaux-nés qui pleurent à leur baptême et montrent tous les signes du refus? Il faut leur demander : pourquoi Dieu ne sauvet-il pas les tout-petits réprouvés, qui n'ont ni voulu, ni refusé leur baptême? Dieu semble plus bienveillant à l'égard des adultes, qu'il veut sauver même s'ils ne le veulent pas, qu'à l'égard des tout-petits qu'il ne veut même pas sauver ${ }^{67}$ !

Cette dernière phrase est d'un humour grinçant, condiment dont Gottschalk assaissonne volontiers ses dialogues. Il lui plaît de ridiculiser l'adversaire, comme le montrent les récits des débats avec Faustus et Halduin. Il manie aussi cette ironie dans la Scedula sur la Trinité, transmise à Hincmar par des clercs de Reims. On lit une note sur l'urbana facetia et la congrua hyronia en marge d'une pointe d'Augustin dans un manuscrit de la Cité de Dieu annoté par un partisan de Gottschalk : l'art des saillies en contexte polémique est soigneusement cultivé par les Carolingiens ${ }^{68}$.

La Scedula sur la Trinité transmise par le De una et non trina Deitate d'Hincmar contient d'emblée un vaste interrogatoire. Quiconque affirme qu'on ne peut pas dire que la déité est trine doit être interrogé : affirme-t-il qu'elle est une naturellement ou personnellement? S'il répond "personnellement», il sera jugé sabellien, avec ceux qui affirment que le Père aussi a souffert la

uelis nolis tandem aliquando credere intellegere confiteri cogeris quod Christus filius dei nullo modo per sanguinem crucis suae redemerit reprobos baptizatos quos non habet reconciliatos neque saluatos perpetim neque mundatos.

66. Ibid., p. 237-8.

67. Ibid., p. 239-40: Interrogandi sunt: Cur ergo saluat paruulos infantes quando baptizantur flentes et quantum possunt reluctantes et omnino baptizari sua propria uoluntate nolentes? Item similiter interrogandi sunt de paruulis reprobis et dicendumest eis: Cur illos uicissim deus non saluat qui sicut nec uolunt ita nec nolunt? Ergo beniuolentiorem deum facitis erga maiores multo utique peiores quia uult eos ipse saluari sed ipsi nolunt, quam erga paruulos sine baptismate morientes quos ipse saluari non uult.

68. Ms. Bourges, BM, 94, f. 40v : Urbana facetia congrua hyronia devant Cité de Dieu, II, 18, 1 (Augustin, s'indignant de l'injuste fin de Regulus, adresse une pointe aux dieux païens : "s'ils n'en rougissent point, c'est alors vrai qu'ils sont en bronze et n'ont pas de sang !»). On trouve une note favorable à la trina deitas de Gottschalk f. 44r, cf. ci-dessous. 
passion ; s'il répond «naturellement », il devra alors affirmer que du point de vue de la personne, elle est trine ; on ne peut pas, en effet, se mettre à changer les hymnes et les livres des Pères en réécrivant trina et una deitas en sancta et una deitas - pique ironique contre Hincmar, qui avait fait interdire la formule trina deitas dans l'hymne Sanctorum meritis inclyta gaudia. Cela aboutit à une catastrophe : voilà qu'on explique aux simplices qu'on ne peut plus dire trina deitas mais seulement una deitas ${ }^{69}$. On trouve encore des traces de débat dans d'autres textes, mais de manière moins explicite ${ }^{70}$.

Ces interrogatoires reflètent-ils la réalité des débats théologiques des années 850 ? Ne s'agit-il pas d'un trope littéraire, qui provoque un effet de source trompeur? Dans la littérature de controverse tardo-antique, le genre des questions-réponses bibliques avait tenu une place majeure ${ }^{71}$. Ces interrogatoires-là manifestaient l'omniprésence du débat théologique dans l'espace public. La thèse classique est qu'à partir du triomphe de l'orthodoxie nicéenne et la disparition des églises concurrentes, ces débats ont disparu. Les interrogationes seraient alors devenues un genre fossile, scolaire, qui ne reflèterait en rien la réalité sociale du débat théologique. Alcuin rédigeait bien des interrogationes qui représentent un simulacre énonciatif, à des fins purement pédagogiques ${ }^{72}$. Cette thèse est le corollaire de la thèse minimaliste vue en introduction ; contrairement aux vastes controverses tardo-antiques et au renouveau hérétique du Moyen Âge central, l'impact social des débats carolingiens serait extrêmement faible ${ }^{73}$.

Comme on l'a vu, un découpage et une lecture appropriés des œuvres de Gottschalk apportent des arguments de poids à la thèse inverse,

69. Lambot, Cuvres théologiques, p. 20.

70. Par exemple ibid., p. 182.

71. Par exemple, dans Augustin, De perfectione iustitiae hominis, 2, 1 et 7, 16.

72. Gustave Bardy, «La littérature patristique des Quaestiones et responsiones sur l'Écriture sainte », Revue biblique, 41, 1932, p. 210-36 et 341-69; et 42, 1933, p. 14-30 (section sur les Carolingiens), 211-29 et 328-353; voir aussi Alex J. Novikoff, The Medieval Culture of Disputation. Pedagogy, Practice, and Performance, Philadelphia, University of Pennsylvania Press, 2013, p. 25-29.

73. En plus de la bibliographie citée en introduction, citons le débat récent sur la fin de la culture du dialogue dans le monde antique : The End of Dialogue in Antiquity, Simon Goldhill ed., Cambridge, Cambridge University Press, 2009 (en particulier «Introduction : Why don't Christians do dialogue ? », p. 1-12), et la réponse d'Averil Cameron, Dialoguing in Late Antiquity, Cambridge Mass., Harvard University Press, 2014. 
maximaliste. Le moine reclus diffuse dans les milieux monastiques nombre de documents relatifs aux controverses sur la prédestination et la Trinité, et même des guides de polémique. Il faut maintenant les inscrire dans leur contexte. Les interrogatoires de Gottschalk peuvent être comparés à d'autres exemples d'interrogatoires en contexte de controverse. Alcuin, à l'occasion de la controverse adoptianiste, adresse à une aristocrate vivant en Septimanie (peut-être Gundrade, cousine de Charlemagne) une mise en garde contre l'hérésie, avec un modèle d'interrogatoire dont voici un passage :

Il faut lui demander ce qu' on trouve entre « vrai » et «non vrai ». S'il répond : « rien », il faut inférer : le Christ, en tant qu'il est homme, est ou bien vrai Dieu, ou bien non. Mais il est parfaitement absurde de dire qu'il est Dieu mais non vrai Dieu. Ou alors, si l'adversaire a peur de le dire, qu'il explique ce qu' on trouve entre « vrai » et «non vrai » selon l'art de la dialectique. Il faut lui demander si une seule personne peut être à la fois un vrai homme et un homme en peinture, c'est-à-dire un homme non vrai. S'il dit que c'est impossible, il faut en déduire : de même, en Christ, qui est une seule personne, il est impossible qu'il y ait en deux natures à la fois du vrai et du non vrai ${ }^{74} \ldots$

Ce texte relève exactement du même genre que les interrogatoires de Gottschalk, mettant en scène des questions introduites par interrogandum est. Comme par une arborescence, on est guidé selon les réponses de l'interlocuteur.

Le livre était alors un support matériel à la discussion, utilisé au plus vif des débats ${ }^{75}$. Un traité inédit rédigé contre une hérésie christologique des années 800-810, niant la résurrection de la chair du Christ, adopte le même mode de discussion et propose à l'adversaire des enchaînements d'arguments étayés par des citations bibliques. Le traité étant inédit, il vaut la peine d'en citer un passage :

74. Epistolae karolini aevi II, Ernst Dümmler ed., Berlin, Weidmann, 1895 («MGH Epistolae » 4), n² 204, p. 337-40 (datation de la lettre en 799 par Wilhelm Heil, Alkuinstudien 1. Zur Chronologie und Bedeutung des Adoptianismusstreites, Düsseldorf, L. Schwann, 1966, p. 44), ici p. 339 : Interrogandum est, quid sit medium inter verum et non verum? Si dicit : nihil, inferendum est : ergo Christus secundum quod homo est, aut verus Deus est aut non verus. Sed valde absurdum est dicere eum Deum esse, sed non verum. Aut si hoc erubescit adversarius dicere, proferat quid sit medium inter verum et non verum. Secundum artem dialecticam interrogandum est si una persona possit esse homo verus et homo pictus, qui est non verus homo? Si dicit, non posse, inferendum est, nec in Christo, qui est una persona in duabus naturis, verum esse potest, et non verum; sed quidquid in eo est, verum est.

75. Cf. Close, Uniformiser la foi, p. 101-6. Alcuin confie aux prédicateurs méridionaux, en 799, son Adversus Elipandum, en leur donnant des instructions précises sur son usage pendant la prédication ; MGH Epistolae 4, n 201, p. 333-4. 
Il faut leur demander d'abord comment [le Christ] aurait pu manger $d u$ poisson rôti et un rayon de miel $(\mathrm{Lc} 24,42)$ s'il n'avait pas eu un véritable corps; ou bien comment les trous des clous faits dans son corps auraient pu se transférer si vite sur un autre corps; ou si l'apôtre Thomas avait bien reconnu son véritable corps en touchant les endroits des clous et de la pointe de lance, lorsqu'il dit : mon Seigneur et mon Dieu! (Jn 20, 28). Il faut encore leur demander : où et quand a été retiré son corps du tombeau où il avait été placé ? Si ce n'était pas son corps véritable, que Thomas a vu transpercé et dans lequel il a reconnu le vrai fils de Dieu, peut-être veulent-ils dire qu'on n'a pas retrouvé son corps au tombeau car il avait pourri, comme le font les corps humains, et s'était décomposé ? Mais le psalmiste leur résiste : Mon corps repose en sécurité (Ps 16,9), et : Tu ne permettras pas que ton bien-aimé voie la corruption (Ps 16, 10), et ceci : Il garde tous ses os (Ps 34, 21) ${ }^{76}$.

L'art du débat est, ici aussi, fort proche de celui de Gottschalk. L'enchaînement des arguments s'articule autour de citations bibliques, forçant progressivement le contradicteur à céder. L'interrogatoire doctrinal correspond certes à un genre littéraire hérité de l'antiquité et ancré dans le contexte scolaire, mais cela ne signifie pas qu'il s'agit d'un genre fossile : il est réactivé pendant les controverses.

\section{LA CULTURE CAROLINGIENNE DU DÉBAT}

Ces preuves de débats oraux appuyés sur le texte biblique appellent des remarques supplémentaires. D'une part, on sait que

76. Ms. Berlin, SBPK, Hamilton 132, f. 250r : Illis uero hereticis qui ita errant sic respondendum est. Interrogandi sunt in primis quomodo potuisse edere de parte piscis assi et de fauo mellis (Lc 24,42$)$ nisi uerum corpus habuisset, uel quomodo tam cito potuissent uulnera clauorum que in eius corpore fuerunt facta in aliud corpus transmutari, aut si apostolus thomas uerum corpus eius tactis locis clauorum et punctu lancee cognouisset, quando dixit : deus meus et dominus meus (Jn 20,28) ? Et illud adhuc inquirendum est : ubi uel quomodo translatum fuisset corpus eius de monumento in quod positum fuerat. Si hoc non fuit uerum eius corpus quod thomas uidit perforatum et uerum cognouit filium dei, forsitan mallunt dicere quod ideo non fuisset inuentum corpus eius in monumentum quia putrescens sicut humana solent corpora ad nihilum fuisset redactum ? Sed illis contrasistit psalmista dicens : caro mea requiescit in spe (Ps 16,9), et iterum: Non dabit sanctum tuum uidere corruptionem (Ps 16, 10 ; cf. Ac 2, 26-7), et illud: os non conteretur ex eo (Ps 34, 21). - Sur ce traité inédit, quasiment aucun travail scientifique n'a encore été accompli. Voir Wilhelm Wattenbach, « Die Handschriften der Hamiltonschen Sammlung », Neues Archiv, 8, 1883, p. 327-46, p. 332-5; Donald Bullough, Carolingian Renewal, Sources and Heritage, Manchester, Manchester University Press, 1991, p. 236, note 139 ; Das Konzil von Aachen 809, Harald Willjung ed., Hannovre, Hahnsche Buchhandlung, 1998 («MGH Concilia» II, Supp. 2), p. 48. 
des débats de cette nature avaient lieu lors des conciles où les questions de dogme étaient discutées. D'autre part, plusieurs textes offrent des témoignages concordant avec les écrits polémiques de Gottschalk quant à la nature du débat théologique.

Dès avant les Carolingiens, il est question de débats publics arbitrés par le roi. On peut mentionner le débat public qui oppose évêques catholiques et ariens sous l'arbitrage du roi wisigoth Reccared en 587, relaté par Grégoire de Tours ${ }^{77}$; ou celui qui oppose Grégoire le Grand, alors apocrysiaire à Constantinople, au patriarche Eutychès au sujet de la résurrection de la chair, débat dans lequel s'immisce Tibère II $(578-82)^{78}$. Ce dernier débat, comme ceux que nous avons décrits, a pour fil directeur un enchaînement de citations bibliques.

Plus on progresse dans le haut Moyen Âge, plus les autorités patristiques entrent en ligne de compte. Il faut citer d'abord le débat qui oppose Alcuin à Félix d'Urgel au concile d'Aix-la-Chapelle de juin 799, dans la phase finale de la controverse adoptianiste. Félix comparait avec un recueil de sententiae pour se défendre. Les yeux penchés sur leurs livres, les deux controversistes rompent lance sur lance, jusqu'à ce que Félix, selon la Vita Alcuini, se voie opposer un passage décisif de Cyrille d'Alexandrie qui le fait plier $^{79}$. En 810, alors que Léon III s'oppose à la volonté de Charlemagne de faire chanter dans les paroisses le credo de Nicée-Constantinople augmenté du filioque, la ratio romana transcrit avec une précision sténographique le débat opposant sur ce sujet le pape et les ambassadeurs francs. La discussion se déroulant sur un terrain pastoral et politique, elle n'est pas structurée par des citations bibliques ${ }^{80}$. C'est encore le cas de

77. Hans-Werner Goetz, « La compétition entre catholiques et ariens en Gaule : les entretiens religieux ("Religionsgespräche") de Grégoire de Tours », in Agôn. La compétition, $V^{e}-\mathrm{XII}^{e}$ siècles, François Bougard, Régine Le Jan, Thomas Lienhard dir., Turnhout, Brepols, 2012 (« HAMA » 17), p. 183-98.

78. Moralia in Job, XIV, 56 : PL 75, col. 1077-80. Paul Diacre, Historia Langobardorum, Georg Waitz ed., Hannovre, Hahnsche Buchhandlung, 1878 («MGH Scriptores rerum germanicarum», 48), p. 122 semble s'inspirer entièrement des Moralia.

79. Cf. la confession de Félix d'Urgel, MGH Epistolae 4, p. 329 ; et le récit de la Vita Alcuini, VII, 13 (PL 100, col. 98).

80. MGH Concilia II suppl. 2, p. 287-94. Voir Peter Gemeinhardt, FilioqueKontroverse zwischen Ost- und Westkirche im Frühmittelalter, Berlin, De Gruyter, 2002, et Edward Siecienski, The Filioque. History of a Doctrinal Controversy, Oxford, Oxford University Press, 2010 (« Oxford Studies in Historical Theology »), 
Gottschalk, qui se rend au concile de Quierzy de 849 avec un florilège, qu'on le contraint à jeter au feu ${ }^{81}$. Contrairement à ce que l'on a parfois écrit, il y eut une réelle discussion doctrinale à Quierzy ${ }^{82}$. Selon Hincmar, Gottschalk a émis in synodo (ce qui ne peut se rapporter qu'à Quierzy) des critiques contre Jean Chrysostome, dont la pensée est résolument plus optimiste que celle d'Augustin sur la prédestination et le libre-arbitre ${ }^{83}$. Enfin, en novembre 849, Prudence de Troyes soumet au synode provincial de Paris un recueil d'extraits patristiques sur la prédestination ${ }^{84}$.

Dans plusieurs cas, le concept juridique de ratio émerge, avec ses composés, pour caractériser la discussion. C'est le cas, bien sûr, de la ratio romana de 810 . Mais on retrouve ce lexique au concile de 799, après lequel Alcuin écrit, au sujet de Felix : rationabili disputatione ou rationabiliter auditus et convictus; sans oublier le concile de Mayence de 848, où, selon les Annales de Fulda, Gottschalk a été rationabiliter convictus. Les composés de ratio ont là certainement le sens non tant de «raison » opposée à «l'autorité » que de procédure jugée conforme et menant à des conclusions sûres. Ainsi, la discussion, si formelle qu'elle puisse être, est la condition sine qua non de la qualification hérétique ${ }^{85}$.

La discussion doctrinale ne se limite pas au cadre conciliaire. On trouve d'autres preuves de ces débats qui se jouaient sur des enchaînements de citations bibliques et patristiques. Hincmar cite, dans son De praedestinatione (859-860), les traités dont se servent les augustiniens pour asseoir leurs thèses: l'Hexameron

p. 87-109; Irene van Renswoude publiera une analyse de la ratio romana dans la livraison 2017 d'Early Medieval Europe.

81. Florus, Liber de tribus epistolis, CCCM 260, p. 370.

82. La thèse du concile joué d'avance remonte aux érudits modernes à sympathies jansénistes, et est encore défendue par Albert Freystedt, «Studien zu Gottschalks Leben und Lehre », Zeitschrift für Kirchengeschichte, 18, 1898, p. 1-22.

83. Voir Hincmar, De praedestinatione, PL 125, col. 139.

84. PL 115, col. 1156.

85. Alcuin, Adversus Elipandus libri quattuor, I, 12 et 16 (PL 101, col. 249 et 252) : rationabili disputatione convictus [...] rationabiliter auditus et veraciter convictus. Felix lui-même écrit qu'il a pu être jugé ratione veritatis, MGH Epistolae 4, p. 329, où il faut entendre que la discussion n'a souffert aucun biais. Les Annales Fuldenses, Friedrich Kurze ed., Hannovre, Hahnsche Buchhandlung, 1891 ( MGH Scriptores rerum germanicarum » 7), p. 38, écrivent rationabiliter ut plurimis visum fuit convictus est. On ne doit pas comprendre ici les frictions entre raison et autorité : Felix lui-même, MGH Epistolae 4, p. 330, dit avoir été convaincu d'hérésie auctoritate veritatis, et ne décrit du débat que les auctoritates qui l'ont vaincu. 
de Bède le Vénérable et l'abrégé des Tractatus in Johannem d'Augustin par Alcuin ${ }^{86}$. Ni l'un, ni l'autre ne sont cités par les auteurs que l'on connaît: Hincmar fait sans doute référence à des débats oraux. De même, il existe des traces de la stratégie de dissémination (la Publizistik, pourrait-on dire ${ }^{87}$ ) qu'a adoptée Gottschalk. Vers 856, Hincmar écrit, dans son traité De una deitate, que Gottschalk, «d'abord en cachette, puis ouvertement », a envoyé «un grand nombre d'écrits à autant de monde qu'il pouvait»; ses «complices et fidèles» viennent d'ailleurs de lui apporter une scedula sur la Trinitée ${ }^{88}$. Une décennie plus tard, en 866 , alors que tout semble perdu pour Gottschalk, un moine, Guntbert, s'enfuit du monastère d'Hautvillers pour porter au nom du Saxon un appel au pape Nicolas I ${ }^{\text {er }}$. Hincmar avertit du danger l'archevêque de Sens Egilon, en partance pour Rome. Guntbert avait correspondu fréquemment avec le reclus et avait été soumis, pour cette raison, à de lourdes pénitences ${ }^{89}$.

Quant à Gottschalk, même ses adversaires le campent en virtuose du débat. Hincmar alerte Nicolas I $^{\text {er }}$ en 864 : si le pape veut se saisir de l'affaire Gottschalk et le juger lui-même, Hincmar ne s'y opposera pas. Mais il devra prendre garde, pour l'escorter jusqu'à Rome, à choisir un homme «catholique, ayant la gravité ecclésiastique, vif et bon connaisseur des Écritures », car Gottschalk est capable de "réciter de mémoire toute la journée, sans reprendre sa respiration », des lieux bibliques et patristiques; il cherche à «attraper» tous ceux avec qui il parle $^{90} ;$ et, enfin,

86. PL 125, col. 98.

87. Karl Mirbt, Die Publizistik im Zeitalter Gregors VII, Leipzig, Hinrichs, 1894.

88. Ibid., col. 473-5 : inde plurima scribere et ad quoscunque potuit primum latenter deinde quantum sibi licuit aperte mittere procuravit. Novissime, diebus istis, hanc subjunctam schedulam quae ad nos communiter per complices ac satellites suos pervenit inde conscripsit. De façon caractéristique, Hincmar emploie le terme de satellites, utilisé dans les sources annalistiques pour désigner des fidèles ou vassaux.

89. MGH Epistolae 8, p. 194-6.

90. Ibid., p. 162: Quem si cuicumque commendandum vestra auctoritas viderit, hoc in commendatione etiam providebit, ut cui commendabitur catholicus sit et gravitatem ecclesiasticam et vigorem ac scripturarum scientiam habeat, quia non solum scripturas ad suum sensum violenter inflexas, sed et catholicorum dicta detruncata per totum diem sine respiratione aliqua praevalet memoriter decantare. Unde non solum idiotas in admirationem sui abducere, verum et sciolos 
s'il n'y parvient pas, il affirmera sans relâche, à grand renfort de serments, que son interlocuteur a dit des choses qu'il n'a pas dites, pour prouver que lui dit la vérité et son contradicteur des mensonges contraires à sa doctrine ${ }^{91}$.

Ne retrouve-t-on pas ici le Gottschalk des interrogationes que nous avons vues plus haut, qui, après avoir acculé son adversaire aux confins d'un labyrinthe de citations bibliques soigneusement commentées, le contraint à confesser les thèses voulues ?

\section{EN MARGE DU MANUSCRIT DE BERNE}

Un dernier témoin des débats que Gottschalk laisse dans son sillage se trouve être le manuscrit Berne 584 lui-même. Si les péripéties qui ont amené un scriptorium à recopier les papiers de Gottschalk en l'état et sans nom d'auteur restent obscures, il ne fait guère de doute qu'un lecteur de la toute fin du $\mathrm{IX}^{\mathrm{e}}$ ou du début du $\mathrm{x}^{\mathrm{e}}$ siècle leur a accordé toute son attention. Des feuillets un à huit, qui contiennent l'opuscule De trina Deitate, le nom des Pères de l'Église et des livres bibliques cités par Gottschalk est noté en marge avec un gamma capitulaire (Cyprien, Augustin...). À ces notes de repérage s'ajoutent de courtes notes de contenu sur les points forts du texte, par exemple au feuillet $7 \mathrm{v}$ : cherubin cum sint multi simul plenitudo singulariter dicuntur; potestas pro deo adesse ; homo potens deus potestas ${ }^{92}$. Plus important : sur le premier feuillet, recto et verso, un court texte a été noté dans la marge de tête et de gouttière. Les dégats causés par l'eau sur ce premier feuillet le rendent impossible à reconstituer intégralement, mais on y lit malgré tout un texte trinitaire ${ }^{93}$. En 1r, on discerne : celsus etiam in masculino genere pro celsitudine [...] pro deitate, medicus pro medicina, homo pro humanitate. L'objet de la note est, à l'évidence, de déduire la formule trina deitas, controversée dans les années 850

et incautos $[\ldots]$ in sententiam suam solitus erat traducere $[\ldots]$ ut secum loquentes vel in sermone capere valeat.

91. Ibid., p. 162-3 : Et si de veritate non poterit, invincibiliter sacramentis affirmare curabit ea secum loquentes dixisse, quae forte non dixerant, ut ipse verax et illi, qui ei contradicunt, probentur esse mendaces et adversus suam doctrinam docentes.

92. Lambot, Euvres théologiques, p. 94-5.

93. Remarqué et transcrit ibid., p. 81. 
et défendue par Gottschalk, de la formule trinus deus, consensuelle. De façon frappante, ce lecteur emploie la même méthode que l'éditeur contemporain d'un exemplaire de la Cité de Dieu, en région rémoise (Bourges, $\mathrm{BM}, 94$ ), qui annote ainsi son manuscrit :

[esclavage] c'est-à-dire esclaves : de même, les offices sont souvent mis à la place des officiers; les services, à la place des serviteurs ; l'humanité, à la place des humains ; et de même, la déité à la place de Dieu ; donc trine déité est parfaitement juste ${ }^{94}$.

Les annotateurs des manuscrits de Bourges et de Berne témoignent d'une part de l'écho du débat sur la trina deitas, dans lequel s'était engagé Gottschalk, d'autre part de la diffusion d'une méthode employée pour justifier cette formule controversée en posant l'équivalence entre l'espèce ou la qualité et l'individu (Dieu pour divinité, haut pour hauteur, médecin pour médecine, homme pour humanité).

Ce texte trinitaire fragmentaire se poursuit dans le manuscrit de Berne, f. $1 \mathrm{v}$ : quoniam in deo nulla est aut esse [potest] pluralitas dicitur de eo [trinitas] non triplicitas, unde a multis reprehensus est Sedulius qui dixit simplex [la suite est illisible] ${ }^{95}$. Cette note révèle un excellent degré d'information de la part de l'annotateur. Le poète Sedulius (que l'on doit bien sûr préférer au contemporain de Gottschalk, Sedulius Scottus) écrit en effet dans le Carmen Paschale, I, v. 298 : quod simplex triplicet, quodque est triplicabile simplet ${ }^{96}$. La citation entière de Sedulius éclaire donc l'annotation : ce n'est pas le terme simplex qui pose problème, mais les termes triplicare et triplicabile en lieu et place de Trinitas, qui donnent le sentiment que la substance unique des trois personnes se divise.

Or, l'annotateur n'est pas le premier à remarquer le passage. Gottschalk ne le cite pas, mais cite à l'appui de sa thèse un autre passage de Sedulius (I, v. 324) où il est question de la terna fides, qu'il ne faut pas comprendre, précise Gottschalk,

94. F. 44r (livre II, chap. 6) : id est servi : sic officia saepe ponuntur pro officialibus ; obsequia pro obsequentibus, humanitas pro hominibus; deitas pro deo ; hinc rectissime trina deitas. Cf. Bernhard Bischoff, Katalog derfestländischen Handschriften des neunten Jahrhunderts (mit Ausnahme der wisigotischen), t. I : Aachen-Lambach, Wiesbaden, Harrassowitz, 1998, p. 143, n 675 : «Wohl Nähe zu Reims, IX. Jh., 3. Drittel ».

95. Lambot, Euvres théologiques, p. 83.

96. Carmen paschale, ed. J. Huemer, Sedulii opera omnia, Vienne, Gerold, 1885 (« Corpus scriptorum ecclesiasticorum latinorum » [CSEL] 10), p. 38. 
comme tres fides ${ }^{97}$. Ce passage du Saxon est réfuté dans le De una deitate où Hincmar aligne une série de passages trinitaires de Prudence, Arator et Sedulius, commentant ce qu'il interprète comme des licences poétiques justifiées par la métrique. Hincmar cite, en fin de série, le vers I, 298 de Sedulius (avec triplicare et triplicabile), ajoutant que ce dernier, «poète vénérable », a été "poussé par les impératifs de la métrique ${ }^{98}$. L'archevêque de Reims insiste là-dessus : " C'est pourquoi le témoignage du fidèle poète Sedulius, qui a dû plier l'interprétation droite de la foi aux lois de la métrique, ne prête aucun secours à l'interprétation honteuse de Gottschalk $»^{99}$. Notons bien que ce dernier n'avait pas commis l'erreur de citer ce vers du poète: Hincmar le mobilise par exagération pour jeter le discrédit sur l'usage de Sedulius en général. On comprend mieux l'expression de l'annotateur du manuscrit de Berne : «c'est pourquoi Sedulius a été critiqué par beaucoup de personnes ». Il s'agit là de critiques récentes, venues en premier lieu d'Hincmar. Loin d'être une réflexion solitaire, l'annotation (rémoise, faut-il le rappeler) illustre la place des poètes chrétiens dans la bataille théologique et s'inscrit dans le sillage des débats qui ont opposé les partisans de Gottschalk et ceux d'Hincmar autour des licences poétiques de Sedulius ${ }^{100}$.

Mentionnons, pour finir, les ajouts que l'on trouve au dernier feuillet du manuscrit de Berne, après les hymnes composées par Gottschalk. Comme l'indique Lambot, on lit au f. 147v le Carmen de diebus aegyptiacis du pseudo-Bède ${ }^{101}$ - un calendrier sur les jours dangereux de l'année qui confine à l'astrologie et n'a certes plus rien à voir avec Gottschalk. Puis on lit en marge de pied, sous le Carmen, un extrait corrompu d'un calendrier hygiénique des jours

97. Scedula de trina deitate, ed. Lambot, CEuvres théologiques, p. 25 (préservé par le De una deitate d'Hincmar, PL 125, col. 560).

98. PL 125, col. 564 : Nam et quod venerandus Sedulius, sicut coactus metri necessitate, trinam fidem et in eodem Paschali opere posuit dicens : Quod simplex triplicet, quodque est triplicabile simplet, beatus Augustinus in libro sexto de Trinitate improbat...

99. Ibidem : Quapropter non succurrit pravo sensui Gothescalci testimonium fidelis poetae Sedulius, qui rectum fidei sensum lege metri constrinxit...

100. Gottschalk mobilise fréquemment Sedulius dans ses scedulae, mais surtout pour des motifs grammaticaux (on peut y voir un reflet de son enseignement passé) : cf. Lambot, Euvres théologiques, p. 553.

101. Alexander Riese, Anthologia latina sive poesis latinae supplementum, t. I, Leipsiz, Teubner, 1894, c. 680a (olim 736), p. 156-7. 
où il ne faut subir aucune saignée ou ne prendre aucun traitement ; le même texte se retrouve dans d'autres manuscrits, et s'inspire du De phlebotomia du pseudo-Bède ${ }^{102}$. Ces instructions se rapportent, selon toute vraisemblance, au bétail. On trouve dans bien d'autres manuscrits savants carolingiens des ajouts de même nature ${ }^{103}$.

Enfin, on lit, sur la languette qui subsiste du dernier feuillet (148r), le texte de deux extraits, l'un de l'Ambrosiaster (commentateur tardo-antique des épîtres pauliniennes) ${ }^{104}$, l'autre de la Collatio Augustini cum Pascentio (dialogue contre les Ariens mettant en scène Augustin et composé en Afrique sous domination vandale $)^{105}$. Il est difficile de savoir si les deux extraits

102. [cave mu] ltum ne in tribus diebus sanguinem minuas aut potionem sumas aut de ansere manducas; viii [calendarum aprilis d] ie lune; intrante augusto die lunae; Exeunte decembre die lunae; tunc venae in homine... Cf PL 90, col. 960 : Plures sunt dies Aegyptiaci in quibus nullo modo nec per ullam necessitatem licet homini vel pecori sanguinem minuere, nec potionem impendere, sed ex his tribus maxime observandi octavo idus april, illo die lunis, intrante augusto ; illo die lunis, exeunte decembri; illo die lunis, cum multa diligencia observandum est, quia omnes venae tunc plenae sunt. On trouve le même texte dans le ms. Londres, British Library, Harley 1772, f. 115v - lui aussi un manuscrit carolingien originaire de Reims.

103. Il arrive que des recettes ou incantations soient notées dans les marges de manuscrits carolingiens : Ivrée, $\mathrm{BC}, 38, \mathrm{f} .7 \mathrm{v}-8 \mathrm{r}$ (prières d'intercession à saint Sigismund pour la guérison d'un tiers) ; Londres, BL, Add. 19725, f. 5, 41, 42v (recettes médicales); Paris, BNF, latin 2773, f. 108-9 (une formule magique et un carmen de nigro caballo pour chasser les vers d'un cheval) ; Paris, BNF, latin 1546 (au dernier feuillet, on lit à nouveau une formule pour chasser les vers d'un cheval) ; Paris, BNF, latin 8508, f. 147v (prières à sainte Tècle et saint Nazaire contre une macule ophtalmique) et 160v (à nouveau contre les vers); Vatican, BAV, pal. lat. 973, f. 128v (recette contre les calculs)...

104. Lambot, Euvres théologiques, p. XIV, écrit «Ambroise»; on sait que les Carolingiens ne faisaient pas la distinction entre l'évêque milanais et le commentateur des épîtres pauliniennes. Le microfilm ne m'a pas permis de vérifier si le feuillet 148 est une languette collée au cahier précédent ou bien le dernier feuillet mutilé du cahier.

105. Je transcris : Sanctus Ambrosius super ad Romanos apostoli epistolam. "Lex enim spiritus vitae in Christo iesu liberavit me a lege peccati et mortis " $(R m 8,2)$. securitatem flagitat homini per gratiam Dei ut de suggestionibus non sit sollicitus diaboli, dummodo spernat illas. Nihil enim homini oberunt apud mortem secundam, quia lex fidei, fidei id est spiritus, liberavit hominem a morte secunda dampnato peccato. Nec obest iam homini quia carni inest peccatum, si modo repugnet illi memor auxilii dei. Sed et coronandus est qui peccati in carne manentes [sic] praemit consilia [cf. Ambrosiaster, In Romanos, PL 17, col. 116]. Augustinus. Qui disponit arguere non faciat unde alium nititur accusare. Non inficiant de verbo simplici plenum quasi de stipula oculum fratris qui suum sautiatum tanquam de immanitate non praevident trabis [cf. Collatio Augustini cum Pascentio Ariano, ed. Hildegung Müller, Dorothea Weber et Clemens Weidmann, Vienne, 
ont un lien avec Gottschalk. Si le premier concerne un point de sotériologie - l'Esprit délivrant du péché que la Loi avait permis de reconnaitre -, il insiste surtout sur les rapports entre chair et esprit et n'est pas cité pendant la controverse. Cela dit, l'épître aux Romains en est un des lieux communs. Quant au second, son découpage en fait un morceau relatif à l'éthique du débat. Il faut débattre sans accuser son contradicteur et sans lui reprocher d'avoir une paille dans l'œil quand on a dans le sien une énorme poutre (cf. Mt 7, 3-5). Le texte de la Collatio a subi là une certaine déformation (voir en note). La «parole simple» (verbum simplex) était, à l'origine, l'homoousios (la consubstantialité du Père et du Fils) qu' « Augustin » défend contre les critiques des ariens, accusés pour leur part d'abuser de termes absents de l'Écriture - d'où la paille (l'homoousios) et la poutre (les textes ariens). Or, dans l'extrait du manuscrit de Berne, faute de contexte et avec un changement de cas qui rattache plenum à oculum au lieu de verbo, on comprend tout autre chose : il ne faut pas reprocher à son contradicteur la simplicité de son langage. Cela ferait tout à fait sens dans un contexte où les thèses de Gottschalk seraient encore débattues, quoique localement et sporadiquement. Est-ce un hasard si le passage qui suit immédiatement celui-là dans la Collatio est cité précisément par Hincmar (comme «saint Augustin dans son livre contre Pascentius ») dans son De una et non trina deitate, écrit contre Gottschalk vers $856^{106}$ ?

Il est donc possible que ces deux extraits aient été sélectionnés dans le sillage des débats causés par Gottschalk, lorsqu'a été aussi noté dans la marge du premier feuillet du manuscrit le texte trinitaire. Le bifeuillet du ms. Paris, BNF, latin 12292, f. 1-2, qui contient la Confessio brevior de Gottschalk, a été rempli progressivement d'extraits patristiques exactement de la même manière - et l'on y retrouve, avec des extraits de Jérôme

Österreichische Akademie der Wissenschaften, 2008 ( « Österreichische Akademie der Wissenschaften, philosophisch-historische Klasse, Sitzungsberichte» 779, «Veröffentlichungen der Komission zur Herausgabe des Corpus der lateinischen Kirchenväter », 24) c. 15, p. 98-101]. La version complète de la seconde citation donne de verbo simplici et ratione fidei pleno quasi...

106. Ibid., p. 100 et 59-60; PL 125, col. 551. Le manuscrit de Berne s'ajoute à la tradition indirecte inventoriée par les éditeurs p. 57-60. 
et Haymon, un extrait de l'Ambrosiaster ( $R m$ 9, 21), cette fois parfaitement lié au thème de la controverse.

\section{Conclusion}

Un découpage approprié des opuscules théologiques de Gottschalk montre que ce dernier dissémine dans le milieu clérical des textes courts et polémiques. Il suit là une méthode qui évoque la Publizistik de l'ère grégorienne décrite jadis par Carl Mirbt : son intention, l'énonciation le montre, est que ses correspondants fassent circuler ses textes. Parmi eux, on trouve de véritables modèles d'interrogatoires articulés sur des citations bibliques, évoquant irrésistiblement les controverses tardo-antiques, mais dont on a vu qu'il existe d'autres exemples altimédiévaux. Les Carolingiens ont pu puiser dans les textes de l'Église tardo-antique les règles du débat doctrinal, en s'inspirant des dialogues hérésiologiques et autres textes polémiques des Pères de l'Église, que l'on copie activement au IX ${ }^{\mathrm{e}}$ siècle. Comme on l'a vu, de véritables débats ont eu lieu lors des conciles de 799 et 849 , sans compter la nébuleuse de discussions qui a entouré les controverses carolingiennes. Ces documents permettent de réévaluer la culture du débat et la publicité des discussions doctrinales, deux siècles avant la querelle des investitures où la Publizistik reconnaît traditionnellement la première manifestation d'une opinion publique et de stratégies de propagande de l'histoire européenne.

La description des œuvres de Gottschalk esquissée ci-dessus et détaillée en annexe n'a pas vocation à épuiser le sujet, bien au contraire. Il y aurait là matière à une vaste enquête d'histoire littéraire, solidement appuyée sur l'analyse codicologique du manuscrit de Berne - bref, à une véritable thèse : cet article ne prétend que lui ouvrir la voie. Entre-temps, on espère qu'il aidera les savants à se repérer dans la «jungle» du manuscrit de Berne et à prendre la mesure des différentes strates qui le composent. On espère surtout qu'il attirera l'attention de savants préoccupés de génétique littéraire. On l'a vu, le manuscrit reflète en partie l'état dans lequel ont été rassemblés les papiers de Gottschalk. Or, on ne sait quasiment rien de ce qu'étaient les «fiches » des auteurs carolingiens - cette strate intermédiaire entre les manuscrits 
de travail annotés, dont plusieurs ont été retrouvés, et l'œuvre ou son brouillon définitif, dont certains ont été retrouvés aussi. Gottschalk, on l'a vu plus haut, a été contraint de jeter au feu, à Quierzy en 849 , un volume d'extraits scripturaires et patristiques. La description qu'en donne Florus évoque certains des segments du manuscrit de Berne : «le livre dans lequel il avait rassemblé pour lui des sentences des Écritures et des saints Pères $»^{107}$. C'est le cas d'autres auteurs. Claude de Turin, dans un passage exhumé par Michael I. Allen, confesse avoir « entre les mains de nombreux extraits » au sujet des épîtres du Nouveau Testament qu'il doit commenter ${ }^{108}$. Jeremy C. Thompson, sur la base de similitudes entre le Liber de tribus quaestionibus et le Collectaneum de Loup de Ferrières, a suggéré qu'il disposait de fiches sur des supports éphémères qu'il remaniait et réorganisait au fil du temps ${ }^{109}$. Les parallèles textuels entre différents textes de Gottschalk (bien souvent entre opuscules théologiques et grammaticaux !) montrent que ce dernier avait une méthode comparable. Espérons qu'elle fera bientôt l'objet d'une étude plus complète et que le volume promis jadis par Lambot verra enfin le jour.

warrenpeze@hotmail.com

107. Florus, Liber de tribus epistolis, Zechiel-Eckes ed., CCCM 260, p. 370 : libellum in quo sententias scripturarum sive sanctorum patrum sibi collegerat...

108. Michael I. Allen, «The Chronicle of Claudius of Turin », After Rome's Fall. Narrators and Sources of Early Medieval History, Essays presented to Walter Goffart, A. Murray, Toronto, University of Toronto Press, 1998, p. 288-319 (299, 302). Cf. MGH Epistolae 4, p. 597 (préface du Commentaire sur les Galates) : De caeteris vero epistolis iam multa in manibus nostris tenentur excerpta, ad quas [...] accedere procurabo.

109. Jeremy C. Thompson, «The Circulation of the Predestinarian Works of Lupus of Ferrières in Valenciennes, Bibliothèque municipale 293 », La controverse carolingienne sur la prédestination: histoire, textes et manuscrits, Paris, Études augustiniennes, à paraître (comparant une paraphrase de l'Enchiridion 16, 61 d'Augustin qui se retrouve dans le Liber de tribus quaestionibus et le Collectaneum). 


\section{ANALYSE DE LA COMPOSITION DES OPUSCULES DE GOTTSCHALK}

\begin{tabular}{|c|c|}
\hline $\begin{array}{l}\text { Composition du texte } \\
\text { Source : Lambot } 1945\end{array}$ & Commentaires \\
\hline 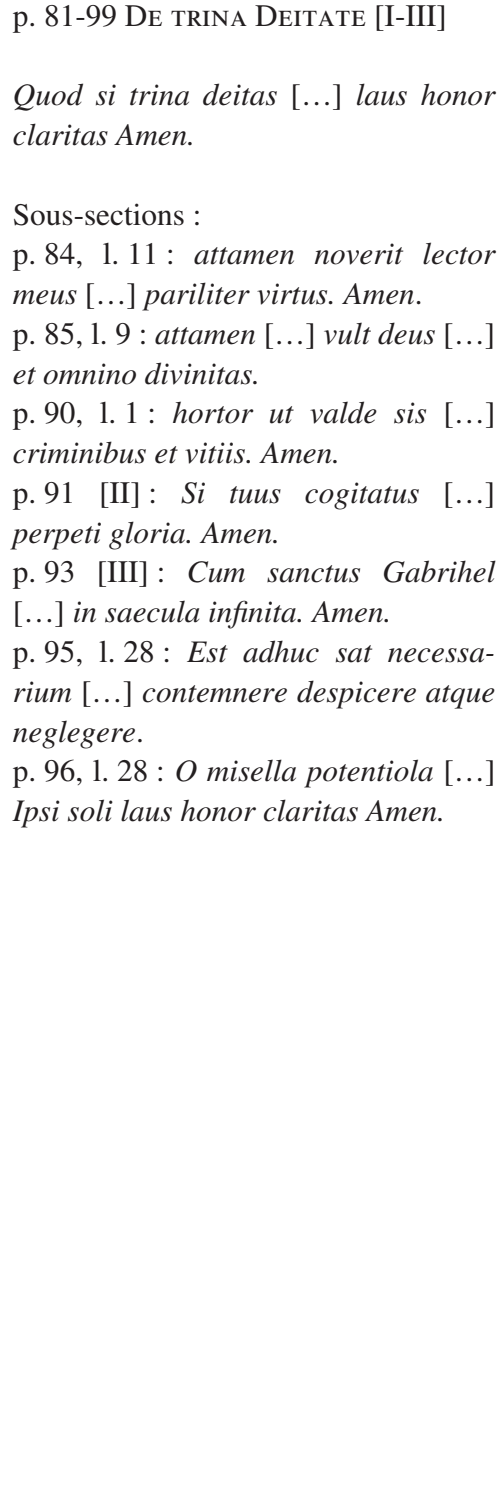 & $\begin{array}{l}\text { Opuscule sur la trina deitas } \\
\text { Remarques : } \\
\text { L'opuscule semble avoir été composé } \\
\text { en plusieurs temps, comme le montrent } \\
\text { les scansions par des explicit, achevés } \\
\text { par des amen (cf. aussi p. xiv). Les } \\
\text { sections ont chacune leur cohérence, } \\
\text { mais forment un enchaînement logique } \\
\text { (cf. p. 90, 1. 3, référence à une citation } \\
\text { de la p. 89, 1. 11 ; p. 93, [III], appro- } \\
\text { fondissement des réflexions esquissées } \\
\text { en fin de [II] sur les prédicats divins; } \\
\text { p. 95, 1. 28 sqq., approfondissement } \\
\text { des réflexions esquissées en [III] sur } \\
\text { la potestas). Elles correspondent donc } \\
\text { aux étapes de rédaction successives du } \\
\text { texte. } \\
\text { Parallèles : } \\
\text { À la réflexion sur la potestas divine } \\
\text { succède, p. 95-96, un enchaînement } \\
\text { politique sur l'obéissance et la déso- } \\
\text { béissance, remployé dans l'opusculum } \\
\text { primum (p. 412) et qui se termine par } \\
\text { une apostrophe à Hincmar (p. 96-99), } \\
\text { sur laquelle se conclut aussi l'opuscule. } \\
\text { Énonciation: } \\
\text { o mi lector veritatis dilector (p. 83) } \\
\text { lector meus (p. 84) sis nunc et istinc } \\
\text { amator (p. 90) vide et cogita (p. 90) } \\
\text { tuus (p. 91) adde (p. 92) cerne mi lector } \\
\text { (p. 94) } \\
\text { intueamur... videbimus... valemus... } \\
\text { obsecremus (p. 95) } \\
\text { Hincmar est invectivé anonymement } \\
\text { (p. 96-99) }\end{array}$ \\
\hline
\end{tabular}




\begin{tabular}{|c|c|}
\hline $\begin{array}{l}\text { Composition du texte } \\
\text { Source : Lambot } 1945\end{array}$ & Commentaires \\
\hline $\begin{array}{l}\text { p. 99-101 ITEM DE TRINITATE [IV] } \\
\text { Sanctus Augustinus dicit }[\text {... }] \text { saecula } \\
\text { infinita. Amen. Amen. Amen. }\end{array}$ & $\begin{array}{l}\text { Scedula courte sur la trina deitas } \\
\text { Pas de destinataire direct. }\end{array}$ \\
\hline $\begin{array}{l}\text { p. 101-130 [EXCERPTA DE TRINITATE, V] } \\
\text { Sanctus Fulgentius dicit [...] } \\
\text { quomodo iudicabitur mundus? }\end{array}$ & $\begin{array}{l}\text { Cette collection d'extraits émane } \\
\text { du même contexte que les scedulae } \\
\text { précédentes. Voir p. } 110 \text { le lien entre } \\
\text { le titre QUID SIT INTER INGENITUM } \\
\text { [...] SED PROCEDENS et l'argumenta- } \\
\text { tion p. } 100 \text {. }\end{array}$ \\
\hline $\begin{array}{l}\text { p. 130-179 [RESPONSA DE DIVERSIS, VI] } \\
\text { i. Quid interest }[\ldots] \text { cuncta de nihilo. }\end{array}$ & $\begin{array}{l}\text { Compilation hétéroclite rassemblée } \\
\text { par Gottschalk (entre autre à partir de } \\
\text { sa correspondance avec des moines), } \\
\text { pourvue d'une table des matières et } \\
\text { adressée à un destinataire de haut rang } \\
\text { (cf. Bouhot 1976, p. 43). Les chapitres } \\
8 \text { à } 16 \text { manquent. }\end{array}$ \\
\hline $\begin{array}{l}\text { p. } 130-132,1.13 \\
\text { i. Quid interest }[\ldots] \text { augmentati remu- } \\
\text { neratorem. Amen. }\end{array}$ & $\begin{array}{l}\text { Table des matières de Gottschalk et } \\
\text { dédicace des quaestiones } \\
\text { Cf. Bouhot 1976, p. } 43 \text {. } \\
\text { Adressé à un dignitaire : vos autem } \\
\text { respondete rectiora (p. 131), fomitem } \\
\text { ingenioli mei succendite (p. 132) }\end{array}$ \\
\hline $\begin{array}{l}\text { p. } 132-138[1-3] \\
\text { Caput i. QUID INTEREST INTER SUBS- } \\
\text { TANTIAM [...] confirmet omni modo. } \\
\text { Amen. } \\
\text { Sous-sections : } \\
\text { p. } 134,1.25: \text { quaero an pater... [2] } \\
\text { p. } 136,1.17: \text { Sicut omnino tibi... [3] }\end{array}$ & $\begin{array}{l}\text { Scedula de réponse à un ami au sujet } \\
\text { de la Trinité } \\
\text { L'homogénéité de la scedula semble } \\
\text { être dégagée par un incipit faisant } \\
\text { référence aux questions du corres- } \\
\text { pondant, et par un amen final. Chaque } \\
\text { question est cependant distinguée par } \\
\text { un alinéa dans le manuscrit, corres- } \\
\text { pondant aux sous-sections ci-contre. } \\
\text { Tutoiement : quaestiones tuas (p. 132) } \\
\text { nanciscaris (p. 137) }\end{array}$ \\
\hline
\end{tabular}




\begin{tabular}{|c|c|}
\hline $\begin{array}{l}\text { Composition du texte } \\
\text { Source : Lambot } 1945\end{array}$ & Commentaires \\
\hline $\begin{array}{l}\text { p. } 138-142,1.18 \text { et p. } 142,1.19-145 \\
\text { [4] } \\
\text { Est vitium nimis noxium }[\ldots] \text { necessa- } \\
\text { rio subaudiretur. Amen. } \\
\text { Sous-sections : } \\
\text { p. } 138: \text { Est vitium nimis noxium... } \\
\text { p. } 142,1.19 \text { : Dubitari nullatenus... }\end{array}$ & $\begin{array}{l}\text { Scedula en deux parties contre la } \\
\text { réforme liturgique d'Halduin d'Haut- } \\
\text { villers (cf. p. 142) } \\
\text { Le titre iiii du sommaire reflète les } \\
\text { deux sous-sections ci-contre (p. 130). } \\
\text { La fin est marquée par amen. } \\
\text { Parallèles : } \\
\text { p. } 138 \text { et } 141 \text { [sous-section p. 138-142] } \\
=\text { p. } 374-375 \text { (opusculum primum) et } \\
\text { p. } 423-424 \text { (opusculum secundum) } \\
\text { Tutoiement: attende et appende et } \\
\text { sponte perpente (p. 142) }\end{array}$ \\
\hline $\begin{array}{l}\text { p. } 145-146[5] \\
\text { Filius propterea }[\ldots] \text { nulla est dissi- } \\
\text { militudo. }\end{array}$ & $\begin{array}{l}\text { Courte scedula sur le fait que le Fils } \\
\text { est l'image du Père } \\
\text { Tutoiement : Lege... cernes... discernes } \\
\text { (p. 146) }\end{array}$ \\
\hline $\begin{array}{l}\text { p. } 146-158[6-7] \\
\text { Liberum arbitrium [...] demersi et } \\
\text { rite... (lacune) } \\
\text { Sous-sections : } \\
\text { p. } 146[6] \text { : Liberum arbitrium... } \\
\text { p. } 149,1.18 \text { : Christe dilecte fili dei... } \\
\text { p. } 150,1.22 \text { : Si quis autem ... } \\
\text { p. } 154[7] \text { : Ceterum sicut verissime... }\end{array}$ & $\begin{array}{l}\text { Collection de textes sur le libre-arbitre } \\
\text { et la prédestination } \\
\text { Les chapitres } 6 \text { et } 7 \text { constituent bien } \\
\text { une même scedula. Plusieurs sous- } \\
\text { sections y sont dégagées par des } \\
\text { alinéas (cf. p. xvi). Ils isolent une } \\
\text { prière adressée au Christ en faveur } \\
\text { du destinataire de la collection } \\
\text { (p. 149-150). L'unité de la collec- } \\
\text { tion est rendue claire par les ceterum } \\
\text { (p. 154-155) et les item (p. 156-158). } \\
\text { Parallèles : } \\
\text { p. } 155-156 \text { (passage sur les syllogismes) } \\
=\text { p. } 206 \text { (De praedestinatione), } 418 \text { et } \\
\text { 459-460 (opusculum secundum). } \\
\text { Tutoiement : legeris (p. 148), vis (151), } \\
\text { lege (p. 152) etc. } \\
\text { Le destinataire est avec entouré par sa } \\
\text { communauté : vobis (p. 155) }\end{array}$ \\
\hline
\end{tabular}




\begin{tabular}{|c|c|}
\hline $\begin{array}{l}\text { Composition du texte } \\
\text { Source : Lambot } 1945\end{array}$ & Commentaires \\
\hline $\begin{array}{l}\text { p. } 159-160,1.16[16] \\
\text { (lacune) quando deus [...] non super- } \\
\text { sunt in illa. }\end{array}$ & $\begin{array}{l}\text { Fin d'une scedula mutilée, consacrée } \\
\text { au pouvoir des démons sur les saints } \\
\text { Tutoiement : Habeas opto (p. } 160 \text { ) }\end{array}$ \\
\hline $\begin{array}{l}\text { p. } 160,1.17-161[17] \\
\text { Quod diabolus }[\ldots] \text { De correptione et } \\
\text { gratia. }\end{array}$ & $\begin{array}{l}\text { Fragment consacré à la prescience des } \\
\text { démons } \\
\text { Tutoiement : Lege (p. 161) }\end{array}$ \\
\hline $\begin{array}{l}\text { p. } 162-164,1.8[17] \\
\text { Si SIBILLAE ET CETERI VATES... [...] } \\
\text { reprobi utriusque sexus. }\end{array}$ & $\begin{array}{l}\text { Scedula sur la prescience des prophètes } \\
\text { païens } \\
\text { Tutoiement : Lege (p. 163), non displi- } \\
\text { cet mihi si vis hinc adhuc nosse tu } \\
\text { magis (p. 163) }\end{array}$ \\
\hline $\begin{array}{l}\text { p. } 164,1.9-165[17] \\
\text { Sicuti sancta scriptura [...] digni } \\
\text { sumus mundo. Amen. }\end{array}$ & $\begin{array}{l}\text { Nouvelle scedula sur le même thème } \\
\text { envoyée quelques jours plus tard } \\
\text { (nuper) } \\
\text { Plusieurs correspondants: quaestioni } \\
\text { vestrae immo quaestionibus vestris } \\
\text { (p. 164) }\end{array}$ \\
\hline $\begin{array}{l}\text { p. } 166-171 \text { (1. 14) [17] } \\
\text { Noveritis [...] dabo gloriam. }\end{array}$ & $\begin{array}{l}\text { Troisième scedula sur ce thème, } \\
\text { quelques jours plus tard } \\
\text { Plusieurs correspondants : } \\
\text { Noveritis (p. 164), quaestionem propo- } \\
\text { sitam habetis (p. 171) }\end{array}$ \\
\hline $\begin{array}{l}\text { p. } 171-179[18-20] \\
\text { Noveris care }[\ldots] \text { de nihilo. }\end{array}$ & $\begin{array}{l}\text { Scedula sur la volonté et l'inspiration } \\
\text { des réprouvés } \\
\text { Remarque : la collection RESPONSA DE } \\
\text { DIVERSIS s'achève sans conclusion et } \\
\text { sans explicit. } \\
\text { Tutoiement : noveris care (p. 171) }\end{array}$ \\
\hline
\end{tabular}




\begin{tabular}{|c|c|}
\hline $\begin{array}{l}\text { Composition du texte } \\
\text { Source : Lambot } 1945 \\
\end{array}$ & Commentaires \\
\hline p. 180-258 De praedestinatione [VII] & $\begin{array}{l}\text { La compilation De praedestinatione } \\
\text { semble avoir été réalisée, au moins } \\
\text { partiellement, par Gottschalk lui-même : } \\
\text { l'exhortation au lecteur p. 212-217 fait } \\
\text { référence à la compilation de témoi- } \\
\text { gnages qui la précède (cf. p. 216, 1. 6-7), } \\
\text { cf. aussi le titre p. } 180 \text { et l'explicit p. } 258 \text {. } \\
\text { Cela étant, le caractère hétéroclite de la } \\
\text { compilation, sans parler des répétitions } \\
\text { (p. } 180=206 \text { ), laisse penser que des } \\
\text { papiers sur la Trinité et la grammaire s'y } \\
\text { sont mêlés dans des circonstances floues } \\
\text { (cf. p. 182-197). À en juger en particu- } \\
\text { lier par les p. } 212-7 \text {, qui en constituent } \\
\text { le cœur, le thème est la volonté de salut } \\
\text { restreinte et la rédemption des réprouvés. }\end{array}$ \\
\hline $\begin{array}{l}\text { p. } 180-181,1.12 \\
\text { Cum scriptura }[\ldots] \text { ad de domine }\end{array}$ & $\begin{array}{l}\text { Scedula sur le mot gradus } \\
\text { Parallèles : } \\
\text { p. } 180=\text { p. } 206,380,479-480 \\
\text { Le texte [1], remployé quatre fois } \\
\text { chez Gottschalk, semble ici lié au } \\
\text { texte [2] par l'enchaînement lexical } \\
\text { gradi-gradus }\end{array}$ \\
\hline p. $181,1.13-182,1.19$ & $\begin{array}{l}\text { scedula polémique sur la rédemption } \\
\text { Destiné à une communauté : } \\
\text { quam [...] cantatis (p. 181) } \\
\text { Indirectement adressé à un adversaire } \\
\text { inconnu : } \\
\text { audiat istud ille... Conticescat itaque } \\
\text { iam mutus latrator... }\end{array}$ \\
\hline $\begin{array}{l}\text { p. } 182,1.20-184,1.4[4-7] \\
\text { Sanctus Augustinus [...] cruciatio. }\end{array}$ & $\begin{array}{l}\text { Série de notes d'exégèse sur la grâce } \\
\text { et la Trinité } \\
\text { Parallèles : } \\
\text { p. } 183[6]=\text { p. } 87\end{array}$ \\
\hline $\begin{array}{l}\text { p. } 184-190[8-9] \\
\text { Quisquis gratia }[\ldots] \text { merito destinandi. }\end{array}$ & $\begin{array}{l}\text { Scedula sur la nature et la grâce } \\
\text { Tutoiement : expectas... attendas } \\
\text { (p. 186) cognoscas (p. 184) }\end{array}$ \\
\hline
\end{tabular}




\begin{tabular}{|c|c|}
\hline $\begin{array}{l}\text { Composition du texte } \\
\text { Source : Lambot } 1945\end{array}$ & Commentaires \\
\hline $\begin{array}{l}\text { p. } 190-191[10] \\
\text { Quod ut planius }[\ldots] \text { litem sacrificem. }\end{array}$ & $\begin{array}{l}\text { Scedula grammaticale } \\
\text { Parallèle = p. } 422-423\end{array}$ \\
\hline $\begin{array}{l}\text { p. } 192-193[11] \\
\text { Expedit valde }[\ldots] \text { redemptione } \\
\text { inventa. }\end{array}$ & $\begin{array}{l}\text { Scedula sur l'élection } \\
\text { Tutoiement : fili dilecte (p. 192) }\end{array}$ \\
\hline $\begin{array}{l}\text { p. 193-194 [12] } \\
\text { Ni post pharisaea convivia [..] } \\
\text { fremunt ipsi iuvenes. }\end{array}$ & $\begin{array}{l}\text { Note grammaticale sur le Carmen } \\
\text { paschale de Sedulius (IV, v. 64) } \\
\text { adressée à un ami de Gottschalk } \\
\text { Tutoiement : a me tibi (p. 193) }\end{array}$ \\
\hline $\begin{array}{l}\text { p. } 194-196[13] \\
\text { Licet ipse desiderem [...] testimonia } \\
\text { tua. }\end{array}$ & $\begin{array}{l}\text { Note grammaticale à un ami } \\
\text { Tutoiement : nolo mireris (p. 194) } \\
\text { Vouvoiement : vos autem bene feceri- } \\
\text { tis (p. 195) }\end{array}$ \\
\hline $\begin{array}{l}\text { p. } 196[14] \\
\text { Praeterea scias }[\ldots] \text { rite proiecto. }\end{array}$ & $\begin{array}{l}\text { Note théologique à un ami } \\
\text { Tutoiement : scias (p. 196) }\end{array}$ \\
\hline $\begin{array}{l}\text { p. } 197[15] \\
\text { Sane plurimum [...] nimium rei. }\end{array}$ & $\begin{array}{l}\text { Note sur la Trinité } \\
\text { Parallèles : } \\
=\text { De diversis, p. 298-300. } \\
\text { Première personne: dicimus; mei } \\
\text { (p. 197) }\end{array}$ \\
\hline $\begin{array}{l}\text { p. 197-200 TESTIMONIA EVANGELIO- } \\
\text { RUM DE PRAEDESTINATIONE... [VIII] }\end{array}$ & $\begin{array}{l}\text { Collection évangélique sur la prédes- } \\
\text { tination } \\
\text { Pas de destinataire dans l'énonciation. }\end{array}$ \\
\hline $\begin{array}{l}\text { p. 200-202 ITEM TESTIMONIA APOS- } \\
\text { TOLI PAULI DE RE PRAEDICTA }\end{array}$ & $\begin{array}{l}\text { Collection paulinienne sur la prédesti- } \\
\text { nation } \\
\text { Pas de destinataire dans l'énonciation. }\end{array}$ \\
\hline
\end{tabular}




\begin{tabular}{|c|c|}
\hline $\begin{array}{l}\text { Composition du texte } \\
\text { Source : Lambot } 1945\end{array}$ & Commentaires \\
\hline 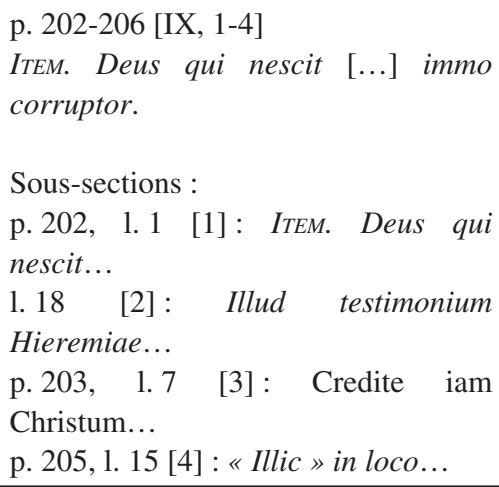 & 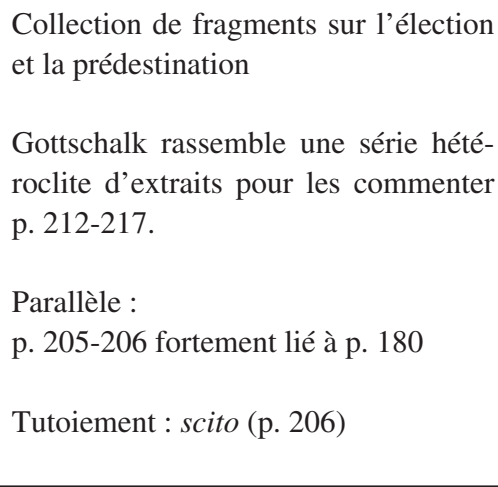 \\
\hline $\begin{array}{l}\text { p. 206-207 De SYLLOGISMIS [5] } \\
\text { Syllogismus est [...] illuc praedesti- } \\
\text { nati. }\end{array}$ & $\begin{array}{l}\text { Scedula sur les syllogismes (concerne } \\
\text { en fait l'élection) } \\
\text { Parallèles : } \\
=\text { Responsa de diversis, p. 156-157; } \\
\text { Opusculum primum, p. } 419 \text {; opuscu- } \\
\text { lum secundum, p. } 459 . \\
\text { Pas de destinataire dans l'énonciation. }\end{array}$ \\
\hline $\begin{array}{l}\text { p. } 207,1.27-209,1.18[6] \\
\text { Erubesce Sidon [...] tunc impletur } \\
\text { istud : Erubesce Sidon, ait enim mare. }\end{array}$ & $\begin{array}{l}\text { Commentaire exégétique sur un verset } \\
\text { d'Isaie } \\
\text { Gottschalk a été interrogé sur ce } \\
\text { verset (Is } 23,4 \text { ) qui est sans rapport } \\
\text { avec le reste du De praedestinatione. } \\
\text { Cette scedula indépendante a pu s'in- } \\
\text { sérer ici par accident. } \\
\text { Parallèle : } \\
\text { p. } 209=\text { Opusculum primum, p. } 419 \\
\text { Tutoiement : vide (p. 208) non mireris } \\
\text { (p. 209) }\end{array}$ \\
\hline
\end{tabular}




\begin{tabular}{|c|c|}
\hline $\begin{array}{l}\text { Composition du texte } \\
\text { Source : Lambot } 1945\end{array}$ & Commentaires \\
\hline 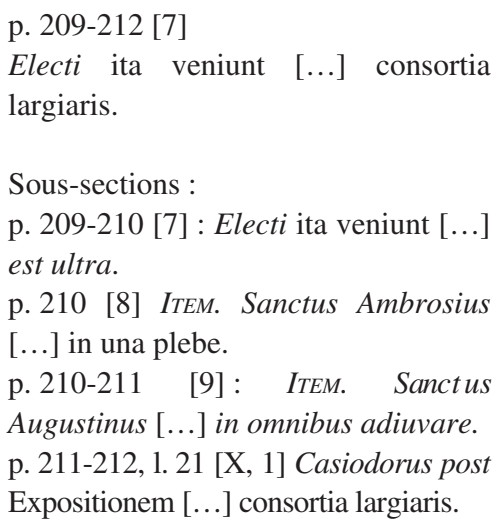 & $\begin{array}{l}\text { Collection patristique sur l'élection et } \\
\text { la rédemption } \\
\text { L'unité des sous-sections est souli- } \\
\text { gnée notamment par l'alinéa dans le } \\
\text { manuscrit (Lambot 1945, p. xvi) et } \\
\text { l'explicit de la première sous-section : } \\
\text { Quid plura? Nihil hinc opus est ultra. } \\
\text { Parallèles : } \\
\text { p. } 209=\text { p. } 227 \\
\text { Tutoiement : teneas... ambigas (p. } 211 \text { ) }\end{array}$ \\
\hline $\begin{array}{l}\text { p. } 212,1.22-217 \\
\text { Haec si cuncta }[\ldots] \text { nihilominus } \\
\text { possidetur. }\end{array}$ & $\begin{array}{l}\text { Conclusion de la collection de frag- } \\
\text { ments et d'extraits précédente au sujet } \\
\text { de l'élection et de la rédemption et } \\
\text { exhortation au lecteur } \\
\text { Remarque : cette exhortation au lecteur } \\
\text { se réfère aux citations qui la précèdent } \\
\text { (p. } 216 \text { : tot superioribus testimoniis). Il } \\
\text { est certain que ces citations embrassent } \\
\text { les p. 209-212, possible qu'elles } \\
\text { embrassent les p. 197-207, mais diffici- } \\
\text { lement envisageable qu'elles remontent } \\
\text { au tout début du De praedestinatione } \\
\text { (p. } 180 \text { ), tant les premiers feuillets sont } \\
\text { désordonnés. De même, il est probable } \\
\text { que celles des scedulae suivantes qui } \\
\text { portent sur le salut des réprouvés (en } \\
\text { particulier p. 218-240) aient été ratta- } \\
\text { chées par Gottschalk lui-même à cette } \\
\text { collection De praedestinatione et que la } \\
\text { succession ordonnée des titres (item de } \\
\text { re praedicta, p. 224) ait été de son fait. } \\
\text { Parallèles : } \\
\text { p. } 217=\text { p. } 208\end{array}$ \\
\hline $\begin{array}{l}\text { p. 218-224 [XI] ITEM CONTRA ILLOS } \\
\text { QUI ADFIRMANT REPROBOS ESSE } \\
\text { REDEMPTOS } \\
\text { Ut fidelis deo }[. . .] \text { in quo requiesci- } \\
\text { mus. }\end{array}$ & $\begin{array}{l}\text { Scedula sur la rédemption des réprouvés } \\
\text { Tutoiement : } \\
\text { attende quae dico } \\
\text { atque consequeris (p. 220) lege (p. 221) }\end{array}$ \\
\hline
\end{tabular}




\begin{tabular}{|c|c|}
\hline $\begin{array}{l}\text { Con } \\
\text { Sou } \\
\end{array}$ & Commentaires \\
\hline $\begin{array}{l}\text { p. 224-231 [XII] ITEM TESTIMONIA DE } \\
\text { RE PRAEDICTA Omnis plantatio [...] } \\
\text { quod est multo amplius. }\end{array}$ & $\begin{array}{l}\text { Collection scripturaire et patristique } \\
\text { sur la rédemption avec un manuel de } \\
\text { questionnement } \\
\text { Tutoiement: sciscitatus fueris... } \\
\text { miraberis (p. 230) }\end{array}$ \\
\hline $\begin{array}{l}\text { p. } 231,1.3-236 \text { [XII-XIII] } \\
\text { REPROBOS NON ESSE REDEMPTOS } \\
\text { CAUTUM EST. Ista qui fideliter [...] } \\
\text { aliena dictauit. }\end{array}$ & $\begin{array}{l}\text { Collection thématique sur la grâce } \\
\text { Tutoiement : non eris (p. 234) } \\
\text { Plusieurs destinataires : } \\
\text { vobis (p. } 232 \text { - peut-être une faute de } \\
\text { copie) }\end{array}$ \\
\hline $\begin{array}{l}\text { p. } 236-240 \text { [XIV] } \\
\text { ITEM DE REPROBIS BAPTIZATIS. } \\
\text { Quantum differant }[\ldots] \text { fiant rei. }\end{array}$ & $\begin{array}{l}\text { Scedula avec interrogatoire sur la } \\
\text { rédemption des réprouvés baptisés } \\
\text { Tutoiement : meministi (p. 237) }\end{array}$ \\
\hline $\begin{array}{l}\text { p. } 240-242[\mathrm{XV}] \\
\text { ITEM. Beatus Augustinus [...] sacrifi- } \\
\text { cabo tibi. }\end{array}$ & $\begin{array}{l}\text { Scedula sur la loi et la grâce inspirée } \\
\text { du De spiritu et littera d'Augustin } \\
\text { Pas de destinataire dans l'énonciation }\end{array}$ \\
\hline $\begin{array}{l}\text { p. } 243-244[\mathrm{XVI}] \\
\text { ITEM. Statera illa }[\ldots] \text { tradidisti te. } \\
\text { Amen. }\end{array}$ & $\begin{array}{l}\text { Explication d'un vers de Job (Jb 31, 6) } \\
\text { Énonciation : prière adressée au Christ }\end{array}$ \\
\hline $\begin{array}{l}\text { p. } 245-247[\mathrm{XVII}] \\
\text { QUARE FILIUS NUNC AEQUALIS NUNC } \\
\text { MINOR PATRE DICATUR. Dominus } \\
\text { noster Iesus Christus }[\ldots] \text { semper } \\
\text { nata divinitas. }\end{array}$ & $\begin{array}{l}\text { Scedula sur l'égalité entre Père et Fils } \\
\text { Remarque : } \\
\text { Cette scedula n'a aucun rapport avec } \\
\text { les textes qui l'entourent. } \\
\text { Pas de destinataire, énonciation ouverte: } \\
\text { Quisquis ut debet attendit (p. 246) }\end{array}$ \\
\hline $\begin{array}{l}\text { p. } 247-254,1.6[\mathrm{XVIII}] \\
\text { ITEM DE ELECTIS ET REPROBIS. Si } \\
\text { super electis }[\ldots] \text { magis ac magis } \\
\text { reus. }\end{array}$ & $\begin{array}{l}\text { Scedula sur la volonté de salut univer- } \\
\text { sel } \\
\text { Tutoiement: } \\
\text { Sicut facile reminisci potes (p. 250) } \\
\text { quaeso te (p. 251) }\end{array}$ \\
\hline $\begin{array}{l}\text { p. } 254,1.7-258 \\
\text { His et talibus testimoniis [...] in } \\
\text { saecula saeculorum. Amen. EXPLICIT } \\
\text { DE PRAEDESTINATIONE. }\end{array}$ & $\begin{array}{l}\text { Extraits non annoncés du De gratia et } \\
\text { libero arbitrio d'Augustin } \\
\text { Fin de la compilation De praedestina- } \\
\text { tione. }\end{array}$ \\
\hline
\end{tabular}




\begin{tabular}{|c|c|}
\hline $\begin{array}{l}\text { Composition du texte } \\
\text { Source : Lambot } 1945\end{array}$ & Commentaires \\
\hline p. 259-279 [DE TRINITATE, XIX] & $\begin{array}{l}\text { Ensemble de Scedulae (p. 259-272, } \\
\text { l. 4) complété par une collection } \\
\text { d'extraits (p. 272-279) sur le caractère } \\
\text { spirituel de la Trinité } \\
\text { Ces scedulae (ou cette scedula) sont } \\
\text { fort difficiles à reconstituer. On peut } \\
\text { faire les constats suivants. Elles ont } \\
\text { été composées l'une après l'autre sur } \\
\text { feuillets séparés. Les incipit détachés } \\
\text { en tête de feuillet dans le Bern } 584 \text { et } \\
\text { les Amen conclusifs à intervalles régu- } \\
\text { liers le laissent penser. Il aurait été } \\
\text { impossible, sans cela, que la scedula } \\
\text { p. 262-264, qui est hors de propos, } \\
\text { s'intercalle entre celle des p. } 259-265 \text {. } \\
\text { Malgré tout, ces scédules ont le même } \\
\text { propos et se font suite comme une } \\
\text { euvre à part entière. La répartition en } \\
\text { scedulae ne regarde que le progrès de } \\
\text { la composition. Peut-être Gottschalk } \\
\text { les a-t-il expédiées au fur et à mesure. }\end{array}$ \\
\hline $\begin{array}{l}\text { p. } 259-262,1.22 \\
\text { Si homo adhaerens domino [...] ac } \\
\text { benignus. Amen. } \\
\text { Sous-sections : } \\
\text { p. } 259-260,1.22 \text { : Si homo adhae- } \\
\text { rens domino }[\ldots] \text { Sufficit deo gratias. } \\
\text { Amen. } \\
\text { p. } 260,1.23 \text { - p. } 262 \text {, } 1.22 \text { : Verum } \\
\text { tamen si quis }[\ldots] \text { ac benignus. Amen. }\end{array}$ & $\begin{array}{l}\text { Scedula incomplète sur le fait que } \\
\text { la Trinité soit composée d'un seul Esprit } \\
\text { Remarque : } \\
\text { Il est possible que l'Amen p. 260, 1. } 22 \\
\text { dégage deux sous-sections, ou qu'un } \\
\text { laps de temps se soit écoulé avant } \\
\text { que Gottschalk reprenne la plume. Il } \\
\text { concluait son premier jet par: suffi- } \\
\text { cit deo gratias (p. } 260,1.21) ; \text { il } \\
\text { commence le second par : si quis [...] } \\
\text { cui totiens repetita disputatio non } \\
\text { sufficiat (p. 260, 1. 23-24). } \\
\text { Tutoiement : intellegas et dicas (p. 260) }\end{array}$ \\
\hline
\end{tabular}




\begin{tabular}{|c|c|}
\hline $\begin{array}{l}\text { Composition du texte } \\
\text { Source : Lambot } 1945 \\
\end{array}$ & Commentaires \\
\hline $\begin{array}{l}\text { p. } 262,1.23-264,1.2 \\
\text { Hinc se sanctus Augustinus }[\ldots] \text { pari- } \\
\text { ter et sacrata. }\end{array}$ & $\begin{array}{l}\text { Scedula sur la sanctification par le } \\
\text { baptême } \\
\text { Remarque : } \\
\text { Cette scedula a été insérée ici par } \\
\text { mégarde. Elle fait exactement la taille } \\
\text { d'un recto. } \\
\text { Pas d'adresse. }\end{array}$ \\
\hline $\begin{array}{l}\text { p. } 264,1.3-265,1.19 \\
\text { Tu vero tene mente }[\ldots] \text { nec agi potest } \\
\text { fructuosius. Amen. }\end{array}$ & $\begin{array}{l}\text { Scedula sur le caractère spirituel } \\
\text { de la Trinité poursuivant celle des } \\
\text { p. } 259-262 \text { (cf. l'incipit p. } 264: \text { Tu } \\
\text { vero... et p. } 265 \text { et cetera cuncta quae } \\
\text { protuli superius : tout cela indique la } \\
\text { continuité). } \\
\text { Remarque: } \\
\text { On trouve p. } 264 \text { une référence à un } \\
\text { autre livre de Gottschalk, manifeste- } \\
\text { ment perdu (cf. note). } \\
\text { Tutoiement : tu tene (p. 264) }\end{array}$ \\
\hline $\begin{array}{l}\text { p. } 265,1.20-269,1.20 . \\
\text { Veraciter intueor } \quad[\ldots] \quad \text { gratuitam } \\
\text { gratiam. Amen. }\end{array}$ & $\begin{array}{l}\text { Scedula sur le caractère spirituel de } \\
\text { la Trinité continuant les précédentes } \\
\text { (cf. le thème donné p. 267). } \\
\text { Remarque : } \\
\text { La distinction avec la scedula précé- } \\
\text { dente est rendue claire par l'exubé- } \\
\text { rance de l'incipit et par sa position } \\
\text { en tête d'un nouveau feuillet (100r). } \\
\text { Gottschalk a visiblement eu une } \\
\text { nouvelle idée et s'est autorisé un } \\
\text { développement supplémentaire, peut- } \\
\text { être quelques jours après avoir écrit } \\
\text { le texte précédent (cf. p. 266: quae } \\
\text { nimis necessaria conspicor haec } \\
\text { supradictis adicere compellor). } \\
\text { Tutoiement: tibi (p. 265), fastidias } \\
\text { (p. 266) etc. }\end{array}$ \\
\hline
\end{tabular}




\begin{tabular}{|c|c|}
\hline $\begin{array}{l}\text { Composition du texte } \\
\text { Source : Lambot } 1945\end{array}$ & Commentaires \\
\hline $\begin{array}{l}\text { p. } 269,1.21-272,1.4 . \\
\text { Ne quaeso frater [...] medullitus } \\
\text { meminerit. Amen. }\end{array}$ & $\begin{array}{l}\text { Scedula sur le caractère spirituel de } \\
\text { la Trinité continuant les précédentes } \\
\text { Remarques : } \\
\text { Une fois encore, un incipit adressé } \\
\text { au lecteur est dégagé sur un nouveau } \\
\text { feuillet (102r) après un Amen. } \\
\text { Gottschalk s'adresse toujours au même } \\
\text { destinataire et fait référence au texte } \\
\text { précédent (p. } 270: \text { apertissime supe- } \\
\text { rius dictus est deus...). } \\
\text { C'est sur cette scedula que s'achève } \\
\text { l'ensemble. L'explicit p. } 271 \text { donne le } \\
\text { thème général. } \\
\text { Tutoiement: } \\
\text { indigneris (p. 269) intellegas... inspi- } \\
\text { cias (p. 271) }\end{array}$ \\
\hline $\begin{array}{l}\text { p. } 272,1.5-279,1.8 \\
\text { Ait enim Augustinus [...] adorandos } \\
\text { vel exorandos. Amen. }\end{array}$ & $\begin{array}{l}\text { Collection d'extraits de la lettre } 238 \\
\text { d'Augustin ajoutée par Gottschalk à la } \\
\text { collection des p. 259-272 } \\
\text { L'explicit p. 278-279 fait référence } \\
\text { à la fois à la série de scedulae et à la } \\
\text { collection d'extraits augustiniens; il } \\
\text { signale la fin - et l'unité - de l'opus- } \\
\text { cule XIX. }\end{array}$ \\
\hline $\begin{array}{l}\text { p. } 279-282 \text { [QUIBUS MODIS DICATUR } \\
\text { REDEMPTIO, XX] } \\
\text { Sicut dicit sanctus Augustinus [...] } \\
\text { repellunt a sua. }\end{array}$ & $\begin{array}{l}\text { Scedula de propagande sur la valeur } \\
\text { rédemptrice du baptême } \\
\text { Remarque : } \\
\text { Le texte est complété par un ajout } \\
\text { sur le caractère spirituel de la Trinité } \\
\text { (p. 282). } \\
\text { Tutoiement du lecteur indéterminé : } \\
\text { Prudens lector attende (p. 281) } \\
\text { Attende benedicte lector (p. 282) }\end{array}$ \\
\hline $\begin{array}{l}\text { p. 283-291 [QUAESTIONES } \\
\text { DE ANIMA, XXI] }\end{array}$ & $\begin{array}{l}\text { opuscule en trois temps (une scedula, } \\
\text { une collection patristique et une } \\
\text { deuxième scedula) sur la question de } \\
\text { l'origine de l'âme }\end{array}$ \\
\hline
\end{tabular}




\begin{tabular}{|l|l|}
\hline \multicolumn{1}{|c|}{$\begin{array}{c}\text { Composition du texte } \\
\text { Source : Lambot 1945 }\end{array}$} & \multicolumn{1}{|c|}{ Commentaires } \\
\hline $\begin{array}{l}\text { p. 283-286, 1. 14 DE ORIGINE ANIMAE } \\
\text { De origine animae sanctus Augustinus } \\
\text { [...] fiat redux. }\end{array}$ & $\begin{array}{l}\text { Scedula dressant un bilan de la } \\
\text { réflexion patristique sur la question de } \\
\text { l'origine de l'âme }\end{array}$ \\
Tutoiement : cautela tua (p. 285)
\end{tabular}




\begin{tabular}{|c|c|}
\hline $\begin{array}{l}\text { Composition du texte } \\
\text { Source : Lambot } 1945\end{array}$ & Commentaires \\
\hline $\begin{array}{l}\text { p. 301-2 [3] } \\
\text { DE INCARNATIONE DOMINI. Hinc } \\
\text { igitur [...] non genus de specie. }\end{array}$ & $\begin{array}{l}\text { Scedula sur les rapports entre le Père } \\
\text { et le Fils } \\
\text { Remploi d'un extrait des opuscules } \\
\text { grammaticaux privé de son incipit: } \\
\text { p. } 313-4 \text { et p. } 478-9 \\
\text { Le titre a été rajouté en marge ; il n'est } \\
\text { peut-être pas voulu par Gottschalk. }\end{array}$ \\
\hline $\begin{array}{l}\text { p. } 302,1.14-17[4] \\
\text { In solis similitudine [...] intellegentia } \\
\text { voluntate. }\end{array}$ & $\begin{array}{l}\text { Fragment sur les analogies de } \\
\text { la Trinité. Il est accolé au fragment } \\
\text { précédent plus loin dans les De diver- } \\
\text { sis, mais pas dans l'opusculum secun- } \\
\text { dum. } \\
\text { Remploi d'un fragment: } \\
\text { p. } 314 \text { et } 482 \text { (opusculum secundum) }\end{array}$ \\
\hline $\begin{array}{l}\text { p. } 302,1.18-304[5] \\
\text { ITEM. Augustinus dicit [...] saeculo- } \\
\text { rum. Amen. }\end{array}$ & Extraits d'Augustin sur la Trinité \\
\hline $\begin{array}{l}\text { p. } 304-9[6] \\
\text { Allocuturus hominem de fide [...] } \\
\text { creavit de nihilo. }\end{array}$ & 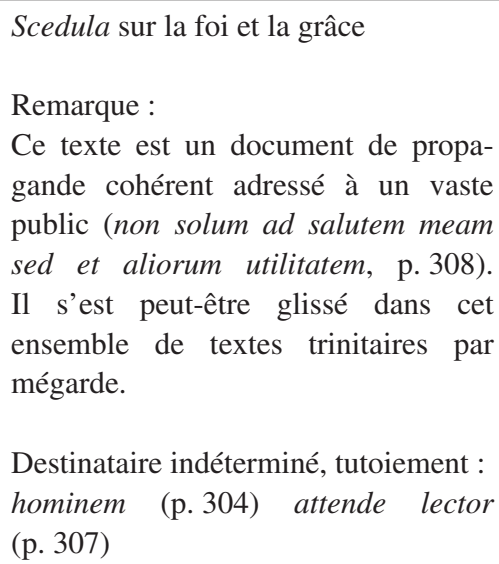 \\
\hline $\begin{array}{l}\text { p. } 309 \text { [7] } \\
\text { Beatus quoque Gregorius [...] sanctus } \\
\text { est. }\end{array}$ & $\begin{array}{l}\text { Fragment sur la Trinité s'insérant dans } \\
\text { une plus vaste collection (quoque) } \\
\text { Parallèle avec l'opusculum secundum } \\
\text { (p. } 460-1 \text { ) } \\
\text { Pas de destinataire }\end{array}$ \\
\hline
\end{tabular}




\begin{tabular}{|c|c|}
\hline $\begin{array}{l}\text { Composition du texte } \\
\text { Source : Lambot } 1945\end{array}$ & Commentaires \\
\hline $\begin{array}{l}\text { p. } 310[8] \\
\text { Pater igitur }[\ldots] \text { in tribus aequalitas. }\end{array}$ & $\begin{array}{l}\text { Fragment sur la Trinité s'insérant dans } \\
\text { une plus vaste collection (igitur) } \\
\text { Parallèle avec l'opusculum secundum } \\
\text { (p. } 468 \text { ) } \\
\text { Pas de destinataire }\end{array}$ \\
\hline $\begin{array}{l}\text { p. } 310[9] \\
\text { Emitte agnum [...] redemit deo. }\end{array}$ & $\begin{array}{l}\text { Court fragment exégétique sur la Trinité } \\
\text { Parallèle avec l'opusculum secundum } \\
\text { (p. 467-468) } \\
\text { Pas de destinataire }\end{array}$ \\
\hline $\begin{array}{l}\text { p. } 310-311[10] \\
\text { Caro et spiritus [...] quantulacumque } \\
\text { dissimilitudo. }\end{array}$ & $\begin{array}{l}\text { Fragment sur les analogies de la Trinité } \\
\text { Parallèle avec l'opusculum secundum } \\
\text { (p. 467-468) } \\
\text { Pas de destinataire }\end{array}$ \\
\hline $\begin{array}{l}\text { p. } 311-313[11] \\
\text { Deus omnipotens }[\ldots] \text { iudicio placere. }\end{array}$ & 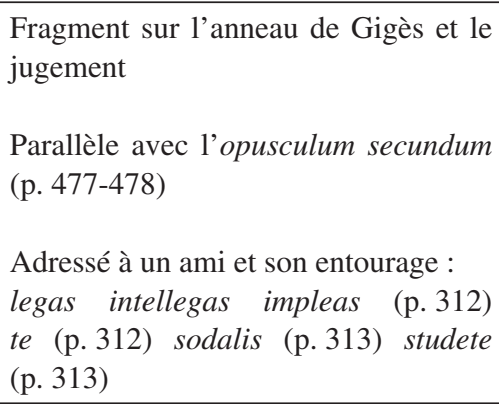 \\
\hline $\begin{array}{l}\text { p. } 313-314[12] \\
\text { Crebro ponitur }[\ldots] \text { non genus de } \\
\text { specie. }\end{array}$ & $\begin{array}{l}\text { Scedula sur les rapports entre le Père } \\
\text { et le Fils } \\
\text { Parallèles : } \\
\text { p. } 301-302 \text { et p. } 478-479 \text { (opusculum } \\
\text { secundum) }\end{array}$ \\
\hline $\begin{array}{l}\text { p. } 314[13] \\
\text { In solis vero similitudine }[\ldots] \text { intelle- } \\
\text { gentia voluntate. }\end{array}$ & $\begin{array}{l}\text { Fragment sur la Trinité employé ici } \\
\text { solidairement avec la scedula précé- } \\
\text { dente (vero), mais pas dans l'opuscu- } \\
\text { lum secundum où il est isolé } \\
\text { Parallèles : } \\
\text { p. } 302 \text { et p. } 482 \text { (opusculum secundum) }\end{array}$ \\
\hline
\end{tabular}




\begin{tabular}{|c|c|}
\hline $\begin{array}{l}\text { Compositio } \\
\text { Source : La }\end{array}$ & Commentaires \\
\hline $\begin{array}{l}\text { p. } 314-316[14] \\
\text { Sicut Paulus apostolus [...] onus } \\
\text { Christi. }\end{array}$ & $\begin{array}{l}\text { Fragment sur saint Paul et la Trinité } \\
\text { Parallèle avec l'opusculum secundum } \\
\text { (p. 482-484) }\end{array}$ \\
\hline $\begin{array}{l}\text { p. } 316-318[15] \\
\text { Item dicit beatus Paulus [...] vocatur } \\
\text { veraciter. }\end{array}$ & $\begin{array}{l}\text { Fragment sur saint Paul et la double } \\
\text { nature du Christ continuant le frag- } \\
\text { ment précédent (item) }\end{array}$ \\
\hline $\begin{array}{l}\text { p. } 318-320[16-19] \\
\text { De deo trinitate }[\ldots] \text { scelerum naevo. }\end{array}$ & $\begin{array}{l}\text { Série de fragments plus ou moins courts } \\
\text { sur la prière à la Trinité (signalés par des } \\
\text { item, p. } 318,1.22 ; 23 ; \text { p. } 319,1.8 ; 19 \text { ). }\end{array}$ \\
\hline $\begin{array}{l}\text { p. } 320[20] \\
\text { De equo }[\ldots] \text { iuvenculum indomitum. }\end{array}$ & $\begin{array}{l}\text { Fragment sur l'analogie entre le } \\
\text { cheval dompté et le croyant }\end{array}$ \\
\hline $\begin{array}{l}\text { p. } 320-324[21] \\
\text { Ipse dominus }[\ldots] \text { in saecula saeculo- } \\
\text { rum. Amen. }\end{array}$ & Longue prière \\
\hline $\begin{array}{l}\text { p. } 324-335 \text { DE CORPORE ET SANGUINE } \\
\text { DOMINI [XXIII] } \\
\text { Quod corpus et sanguis domini [...] } \\
\text { ad gloriam perducat. Amen. }\end{array}$ & $\begin{array}{l}\text { Traité sur l'eucharistie contre Paschase } \\
\text { Radbert }\end{array}$ \\
\hline $\begin{array}{l}\text { p. } 335-337 \text { ITEM DE CORPORE ET } \\
\text { SANGUINE DOMINI } \\
\text { Aliud specialiter corpus domini [...] } \\
\text { corpus et sanguinem domini. }\end{array}$ & $\begin{array}{l}\text { Scedula sur l'eucharistie liée au traité } \\
\text { précédent dans un manuscrit d'Héri- } \\
\text { ger de Lobbes }\end{array}$ \\
\hline $\begin{array}{l}\text { p. 338-346 [DE PRAEDESTINATIONE, } \\
\text { XXIV] }\end{array}$ & $\begin{array}{l}\text { Série de trois textes de dimensions } \\
\text { inégales mais formant un ensemble } \\
\text { cohérent sur la prédestination double } \\
\text { et la rédemption des réprouvés }\end{array}$ \\
\hline
\end{tabular}




\begin{tabular}{|c|c|}
\hline $\begin{array}{l}\text { Composition du texte } \\
\text { Source : Lambot } 1945 \\
\end{array}$ & Commentaires \\
\hline $\begin{array}{l}\text { p. } 338-344[1] \\
\text { At assuescentibus [...] unius mundi, } \\
\text { non duorum? }\end{array}$ & $\begin{array}{l}\text { Scedula sur la gemina praedestina- } \\
\text { tio amputée de son commencement } \\
\text { (commence par at) } \\
\text { Remarque: } \\
\text { Il s'agit d'une réponse à plusieurs objec- } \\
\text { tions sur la double prédestination : que } \\
\text { la prédestination ne soit pas propre à } \\
\text { Dieu ; que le terme « géminé » implique } \\
\text { une pluralité de prédestinations ; que la } \\
\text { prédestination soit le Fils de Dieu; que } \\
\text { la prédestination soit ex nihilo. } \\
\text { Le destinataire a l'estime de Gottschalk } \\
\text { (sans doute pas Hincmar, comme } \\
\text { l'indique Lambot en note p. } 340 \text {; les } \\
\text { arguments sont bien différents - et plus } \\
\text { compliqués - de ceux avancés d'habi- } \\
\text { tude par l'archevêque) : Haec si velut } \\
\text { disertitudini tuae conducit animositate } \\
\text { postposita, consideres... (p. } 340-341 \text { ) }\end{array}$ \\
\hline $\begin{array}{l}\text { p. } 344[2] \\
\text { Sanctus Athanasius [...] reliquerunt. }\end{array}$ & $\begin{array}{l}\text { Fragment sur le verset des trois } \\
\text { témoins (1 Jn 5, 7-8) } \\
\text { Remarque : } \\
\text { Ce court fragment est en rupture } \\
\text { syntaxique avec le texte précédent, } \\
\text { quoique son argument soit le même } \\
\text { (le baptême ne sauve pas les réprou- } \\
\text { vés). Il peut s'agir d'un item rajouté à } \\
\text { l'appui de la thèse. }\end{array}$ \\
\hline $\begin{array}{l}\text { p. } 344-346[3] \\
\text { Item dicit beatus Paulus }[\ldots] \text { nec redi- } \\
\text { mendos. }\end{array}$ & $\begin{array}{l}\text { Scedula sur le baptême des réprouvés } \\
\text { Remarque : } \\
\text { Peut être la suite de la Scedula } \\
\text { p. } 338-344 \text {, dont la fin était consacrée } \\
\text { à cette question. }\end{array}$ \\
\hline $\begin{array}{l}\text { p. } 346-350 \text { [HIERONYMUS IN EZECHIE- } \\
\text { LEM DE RESURRECTIONE MORTUORUM, } \\
\text { XXV] } \\
\text { Famosa est visio }[\ldots] \text { Israhel sociorum } \\
\text { eius. }\end{array}$ & $\begin{array}{l}\text { Extrait de l'In Ezechielem, XI, } 37 \text { de } \\
\text { Jérôme sur la résurrection (peut-être } \\
\text { en lien avec les réflexions sur la résur- } \\
\text { rection de la Scedula précédente) }\end{array}$ \\
\hline
\end{tabular}

